\title{
Background Radiance Estimation for Gas Plume Quantification for Airborne Hyperspectral Thermal Imaging
}

\author{
Ramzi Idoughi, ${ }^{1,2}$ Thomas H. G. Vidal, ${ }^{1}$ Pierre-Yves Foucher, ${ }^{1}$ \\ Marc-André Gagnon, ${ }^{3}$ and Xavier Briottet ${ }^{1}$ \\ ${ }^{1}$ ONERA, The French Aerospace Lab, DOTA, 2 Avenue Edouard Belin, 31400 Toulouse, France \\ ${ }^{2}$ Institut Supérieur de l'Aéronautique et de l'Espace (ISAE), Toulouse, France \\ ${ }^{3}$ Telops Inc., 100-2600 St-Jean-Baptiste Avenue, Québec, QC, Canada G2E 6J5
}

Correspondence should be addressed to Pierre-Yves Foucher; pierre-yves.foucher@onera.fr

Received 2 October 2015; Accepted 23 February 2016

Academic Editor: Hassen Aroui

Copyright (C) 2016 Ramzi Idoughi et al. This is an open access article distributed under the Creative Commons Attribution License, which permits unrestricted use, distribution, and reproduction in any medium, provided the original work is properly cited.

\begin{abstract}
Hyperspectral imaging in the long-wave infrared (LWIR) is a mean that is proving its worth in the characterization of gaseous effluent. Indeed the spectral and spatial resolution of acquisition instruments is steadily decreasing, making the gases characterization increasingly easy in the LWIR domain. The majority of literature algorithms exploit the plume contribution to the radiance corresponding to the difference of radiance between the plume-present and plume-absent pixels. Nevertheless, the offplume radiance is unobservable using a single image. In this paper, we propose a new method to retrieve trace gas concentration from airborne infrared hyperspectral data. More particularly the outlined method improves the existing background radiance estimation approach to deal with heterogeneous scenes corresponding to industrial scenes. It consists in performing a classification of the scene and then applying a principal components analysis based method to estimate the background radiance on each cluster stemming from the classification. In order to determine the contribution of the classification to the background radiance estimation, we compared the two approaches on synthetic data and Telops Fourier Transform Spectrometer (FTS) Imaging Hyper-Cam LW airborne acquisition above ethylene release. We finally show ethylene retrieved concentration map and estimate flow rate of the ethylene release.
\end{abstract}

\section{Introduction}

Anthropogenic sources, especially industrial, have a major contribution to air pollution and security issues. However, these emissions remain poorly estimated at a high spatial resolution over heterogeneous scenes, like industrial plants.

Most of these emissions present a spectral signature in the thermal infrared domain. This is the reason why thermal hyperspectral imaging systems are deployed for their characterization. More, as such plume has a small extent they require a high spatial resolution imagery which can be achieved with existing airborne systems [1-3].

However, signature of gaseous effluents differs highly from usual targets since the plume modifies the spectral signature of the background: different pixels in the data cube that contain the same gaseous plume could have a totally different spectral signature. At first order, spectral signature of these pixels will correspond to background spectra affected by either absorption or emission of the gas. This variability depends on background materials and temperature difference between the ground and the plume.

Existing approaches which use the spectral information of such sensor to characterize gas plume can be divided into two stages: endmember decomposition techniques to estimate the background properties [4-6] and trace element detection [7-10] or quantification methods [11-13] based on estimated gas differential signature (difference for each "onplume pixel" between measured radiance and "off-plume" estimated radiance). Then it has been shown that background uncertainty and heterogeneity are one of the major sources of incertitude in gas quantification and detection limit [1416]. Depending on the ground properties the same amount 
of gas can have a signature; thus a retrieved concentration is modified by a factor of ten.

This paper aims to reduce the quantification error due to misestimation of background properties.

To deal with ground variability for heterogeneous scene, clustering was suggested to compute covariance matrices of different spatial classes composing the background [7, 11], thus creating independent classes on which detection algorithms are applied.

The major limitation of these methods is that clustering background step may be contaminated by the presence of the gas plume $[4,5,17]$.

If existing in the reflective domain, for example, the very weak gas plume signature has little impact on the clustering stage $[7,8]$. But for quite strong signature in the thermal domain, gases plume anomalous classes can appear.

To overcome such issues, the clustering phase should be achieved outside the spectral bands corresponding to the strong absorption of the gas to be detected. Some recent works [18] show that such Selected-Band approach can be used to estimate background radiance under the plume with an interesting precision for various scenes.

In this paper, we look for a background estimation method that will efficiently reduce the gas retrieval quantification error in the case of heterogeneous scene. In this work we introduce a novel method to estimate pixel by pixel the offplume radiance under the plume and the specific additional spectral signature introduced by the gas plume using airborne hyperspectral data.

This method aims to overcome the difficulties that arise from the inherent mixture of the heterogeneous background and the gas plume spectra. We decompose the scene according to the spectral-spatial information, and, for each pixel of each cluster, we estimate differential gas signature taking into account background variability and gas spectral behavior.

This paper is structured as follows. Section 2 is dedicated to the description of the methodology. Then, Sections 3 and 4 evaluate the application of our method on synthetic data and Telops Hyper-Cam airborne platform data. Finally, concluding remarks are given in Section 5.

\section{Methodology}

2.1. Plume Detection. Plume detection is an important step for gas quantification process. It allows selecting the plume area, where the background radiance has to be estimated, and the off-plume area useful to getting background properties.

In the literature, several algorithms have been proposed to detect the presence of gas from hyperspectral data. Spectral Matched Filter (SMF) [4] was chosen in this study because it produced a good detection of the plume with a low number of false alarms.

Let $\mathbf{X}$ be a hyperspectral image of $N$ pixels and $N_{\mathrm{B}}$ bands, represented by an $N \times N_{\mathrm{B}}$ matrix. With $\mathrm{T}$ being the gaseous target spectrum, we can write for the $i$ th pixel $\mathbf{x}_{i}$ of the hyperspectral image $\mathbf{X}$ :

$$
\mathbf{x}_{i}=\mathbf{T} \cdot \boldsymbol{\alpha}_{i}+\mathbf{d},
$$

where $\boldsymbol{\alpha}$ is the target fit coefficients (abundances) and $\mathbf{d}$ is the residual vector to be minimized. To retrieve the abundances $\boldsymbol{\alpha}$, we need to solve the unconstrained regression (see (1)). The analytic solution of this regression is

$$
\boldsymbol{\alpha}_{i}=\frac{\mathbf{T}^{T} \boldsymbol{\Sigma}_{\mathbf{X}}^{-1} \mathbf{x}_{i}}{\mathbf{T}^{T} \boldsymbol{\Sigma}_{\mathbf{X}}^{-1} \mathbf{T}}
$$

where $\Sigma_{X}$ is the covariance matrix of this scene. An unbiased estimation of $\boldsymbol{\Sigma}_{\mathbf{X}}$ can be calculated as follows:

$$
\boldsymbol{\Sigma}_{\mathbf{X}}=\frac{1}{N-1} \cdot \sum_{i=1}^{N}\left(\mathbf{x}_{i}-\langle\mathbf{x}\rangle\right)\left(\mathbf{x}_{i}-\langle\mathbf{x}\rangle\right)^{T} .
$$

We consider here the targeted gases as known. Hence, we use the spectra of targeted gases from the Pacific Northwest National Laboratory (PNNL) absorbance Library $[19,20]$ to construct $\mathbf{T}$. The detection performance can be improved by using an estimation of $\boldsymbol{\Sigma}_{\mathbf{X}}$ involving only plume-absent pixels.

Once the vector of abundances $\boldsymbol{\alpha}$ is calculated, the plume mask is deduced by thresholding.

2.2. Selected-Band Approach (SB). The principal components analysis (PCA) was applied by many authors $[10,11,21]$ in order to obtain a representation of the background radiance of plume-present pixels $\left(L_{\mathrm{bkg}}(\lambda)\right)$. First, the principal vectors are computed using the plume-absent pixels radiances. Then, background radiances of plume-present pixels are assumed to lie in the subspace spanned by these principal vectors.

Niu et al. [18] go even further by developing an approach to estimate the background radiance. This approach is based on the fact that, in plume presence, the on-plume radiance $\left(L_{\text {on }}(\lambda)\right)$ is essentially equal to its background (off-plume) radiance over the most transparent spectral bands. Using this observation, they combine a Selected-Band algorithm to determine these transparent spectral bands with the PCA in order to estimate $L_{\mathrm{bkg}}$.

Thereafter, in the present paper, we shall refer to this method simply as Selected-Band (SB).

After a plume detection on the hyperspectral image $\mathbf{X}$, $N_{\text {off }}$ pixels do not contain any plume gas. A $N_{\text {off }} \times N_{B}$ matrix $\mathbf{L}_{\text {off }}$ is constructed by arranging the off-plume measured radiance spectra. A PCA model is then given by

$$
\mathbf{L}_{\text {off }}=\mathbf{U}_{\text {off }} \cdot \mathbf{P}^{\mathrm{T}}+\mathbf{E}_{\text {off }} \text {, }
$$

where $\mathbf{U}_{\text {off }}$ is an $N_{\text {off }} \times N_{p}$ matrix of coefficients for plumeabsent pixels, $\mathbf{P}$ is an $N_{\mathrm{B}} \times N_{\mathrm{p}}$ of principal components, and $\mathbf{E}_{\text {off }}$ is the residual matrix. $N_{\mathrm{p}}$ is the number of considered principal components. For the used data, we found $N_{\mathrm{p}}=10$ to be an appropriate choice. It allows us to save more than $99.9 \%$ of the information.

Since the background radiances of plume-present pixels are assumed to lie in the subspace spanned by the principal components $\mathbf{P}$, the matrix $\mathbf{L}_{\mathrm{bkg}}$ of the background radiance spectra of the $N_{\text {on }}$ plume-present pixels could be expressed as follows:

$$
\mathbf{L}_{\mathrm{bkg}}=\mathbf{U} \cdot \mathbf{P}^{\mathbf{T}}+\mathbf{E}_{\mathrm{bkg}} \cdot
$$


U is an $N_{\text {on }} \times N_{\mathrm{p}}$ matrix of unknown coefficients to be determined. $\mathbf{E}_{\mathrm{bkg}}$ is the residual matrix in this case.

For this purpose, a second PCA model is written for the $N_{\text {on }}$ plume-present pixels, using only the $N_{\mathrm{SB}}$ bands, where the gas absorption coefficient is virtually nil:

$$
\mathbf{L}_{\mathrm{bkg}}^{\mathrm{sb}}=\mathbf{L}_{\mathrm{on}}^{\mathrm{sb}}=\mathbf{U} \cdot \mathbf{P}^{\mathrm{sb}^{\mathrm{T}}}+\mathbf{E}_{\mathrm{on}},
$$

where $\mathbf{L}_{\text {on }}^{\text {sb }}$ is an $N_{\text {on }} \times N_{\mathrm{SB}}$ matrix, containing the on-plume measured radiance spectra for the selected bands. $\mathbf{P}^{\mathrm{sb}^{\mathrm{T}}}$ is an $N_{\mathrm{SB}} \times N_{\mathrm{p}}$ matrix of principal components; $\mathbf{E}_{\mathrm{on}}$ is the residual matrix.

By using the Moore-Penrose pseudoinverse of the matrix $\mathbf{P}^{\mathrm{sb}}\left(\mathbf{P}^{\mathrm{sb}}{ }^{\dagger}=\left(\mathbf{P}^{\mathrm{T}} \mathbf{P}\right)^{-1} \mathbf{P}^{\mathrm{T}}\right)$, a least-square estimate for $\mathbf{U}$ is given by

$$
\mathbf{U}=\mathbf{L}_{\mathrm{on}}^{\mathrm{sb}} \cdot \mathbf{P}^{\mathrm{sb}^{\dagger \mathrm{T}}} .
$$

An estimation of the background radiances of plume-present pixels is thus obtained by the following expression:

$$
\mathbf{L}_{\mathrm{bkg}}=\mathbf{L}_{\mathrm{on}}^{\mathrm{sb}} \cdot \mathbf{P}^{\mathrm{s}^{\dagger{ }^{\mathrm{T}}}} \cdot \mathbf{P}^{\mathrm{T}} .
$$

The SB method has two leading limitations: the first comes from the assumption that $\mathbf{L}_{\mathrm{bkg}}^{\mathrm{sb}}=\mathbf{L}_{\mathrm{on}}^{\mathrm{sb}}$. This equality is not effective if the temperature of the plume is different from the ambient atmospheric temperature. Indeed, this temperature difference will have an impact on the transmission of atmospheric gases and therefore on the on-plume radiance outside gases signatures.

The second limitation relates to the case of heterogeneous soil. SB method as presented by Niu et al. can be limited if some background plume-present pixels are not well represented in plume-absent pixels. We propose otherwise to solve this second point using a classification of the scene.

\subsection{Clustering-Based Selected-Band Method (CSB Method)}

2.3.1. Classification of the Scene. Since Funk et al. [7] the classification of a hyperspectral scene is a main preliminary step of plume characterization through this scene. Funk et al. showed that classification improves the results of plume detection algorithms. They concluded that clustering data and removing the mean values could be seen as a type of automatic background suppression.

Moreover, the classification of the scene aims to carry off the disturbances on plume's quantification due to the heterogeneity of the soil [15].

In this subsection, we explain the classification process in order to enhance the background radiance estimation. A first classification is carried out on the plume-present pixels (a plume detection algorithm is executed upstream); another is done on the rest of the image.

This classifications is organized in two stages: first, we perform a principal components transform [4] on the radiance spectra of the pixels of interest. Then, we just select the first components for further classification because the important background information is included in these components.
Finally, we carry out a classical $k$-means on these components to classify the scene.

The interest of this dimension reduction is to shorten the time processing of the $k$-means algorithm, without deteriorating its performances. The number of the retained components depends on the image to be treated. Usually, the use of the 3 first principal components is sufficient to have a good classification of the scene.

We choose the $k$-means algorithm for our classification, at one hand, because it is an unsupervised algorithm. Thus, it will be appropriate for a large number of different hyperspectral images. On the other hand, this algorithm is one of the fastest clustering algorithms.

At the end of this step, we obtain $N_{C}$ classes in the area without plume and $N_{\mathrm{CP}}$ classes containing plume. The number of these clusters depends on the threshold $D_{\max }$, used to specify the maximum distance of a pixel to the centroid of its cluster.

\subsubsection{Clustering-Based (CB) Background Radiance Estima-} tion. A first approach of Clustering-Based (CB) background radiance estimation we used consists in a classes matching between the plume-present and plume-absent regions.

The area under the plume has a petty spatial extent compared to the rest of the scene, whereof it only contains a small number of classes. In most cases it stands to reason to assume the classes under the plume as an extension of classes outside the plume and we assume that the presence of gas does not change the main properties of background. Indeed, the threshold $D_{\max }$ is selected such that pixels belonging to the same class have nearly the same characteristics (ground temperature and emissivity, atmospheric profiles). Thus, within a class, the off-plume radiance $L_{\text {off }}$ is very close from one pixel to another.

For each plume-present class $\mathrm{CP}_{i}$, the average spectrum of its pixels is calculated; it is then compared to the average spectra of all plume-absent classes $\left(C_{j}\right)_{1 \leq j \leq N_{C}}$. Readers will note that the comparison is performed only on bands where the target gases absorption coefficients are very small. Class $\mathrm{C}_{j *}$ with the minimal distance from the plume-present class $\mathrm{CP}_{i}$ and the latter are considered to be the same. For a pixel $\mathbf{x}_{\mathrm{p}}$ in this class $\mathrm{CP}_{i}$, we consider that its background radiance $L_{\mathrm{bkg}}\left(\mathbf{x}_{\mathrm{p}}\right)$ is equal to the mean radiance of pixels belonging to class $\mathrm{C}_{j *}$.

The CB method bypasses the soil heterogeneity limitation of the method SB. However, it does not take into account the intraclass variability of the background radiance. In the following paragraph we will present another ClusteringBased method that appropriates the benefits of SB and CB methods of $L_{\mathrm{bkg}}$ estimation, without their shortcomings.

\subsubsection{Clustering-Based Selected-Band Method (CSB Method).} In order to improve the background radiance estimation given by the Selected-Band approach (see Section 2.2) and the Clustering-Based method (see Section 2.3.2), we propose to perform this method on each cluster stemming from the classification (Section 2.3.1). 
After a plume detection, classifications of the scene are realized separately for plume-absent pixels and for plumepresent pixels; then each class of plume-present pixels is associated with a plume-absent pixels class. For each plumepresent class $\mathrm{CP}_{i}$, the Selected-Band approach is performed using (8) with a matrix $\mathbf{P}_{\mathrm{C}_{j *}}^{\mathrm{T}}$ derived from a PCA involving the pixels of the class $\mathrm{C}_{j *}$. Recall that the latter corresponds to the plume-absent class with the minimal distance from the plume-present class $\mathrm{CP}_{i}$ :

$$
\mathbf{L}_{\mathrm{bkg}}^{\mathrm{CP}_{i}}=\mathbf{L}_{\mathrm{on}}^{\mathrm{sb} \mathrm{CP}} \cdot \mathbf{P}_{\mathrm{CP}_{i}}^{\mathrm{sb}^{\dagger \mathrm{T}}} \cdot \mathbf{P}_{\mathrm{CP}_{i}}^{\mathrm{T}} \cdot
$$

In the following sections, we will present the improvement of background radiance estimation for plume-present pixels due to this new method, both on synthetic and real scenes.

\section{Application on Synthetic Data}

3.1. Presentation of the Synthetic Data. To evaluate the performances of background radiance estimation, synthetic data of an industrial scene were used. These data were simulated using a tool we have developed in order to provide a comprehensive test where the "truth" is known.

The dimensions of the simulated images are $200 \times$ $200 \times 107$ (rows by columns by spectral dimension). The wavenumber range used is 800 to $1330 \mathrm{~cm}^{-1}$ with a resolution of $5 \mathrm{~cm}^{-1}$. In this subsection, we describe the process of synthetic data generation.

3.1.1. Ground Simulation. We first defined the distribution of soil composition. Several materials frequently present in industrial scenes (asphalt, copper, aluminum, grass, etc.) were chosen (see Figure 1). Then we attributed for each pixel of the image the appropriate physical properties (emissivity and temperature), according to the present material.

The emissivities were taken from ASTER data base [22]. Figure 2(b) represents the emissivities of the different materials present through the synthetic scene.

The ground temperature varies widely depending on weather conditions, soil composition, orientation of the surfaces, and the relief of the scene (shadow effect). It is therefore very difficult to have a realistic estimate of this quantity. The values we have chosen are close to those measured during the CAPITOUL [23] experiment.

In order to take into account the intraclass variability of the ground temperature, a Gaussian white noise was added to this parameter with a standard deviation of 1-3 K according to the material. In contrast, we did not take into account the intraclass variability of ground emissivity.

3.1.2. Atmospheric Profiles Integration. We assume that the scene dimensions are small enough to consider a spatially uniform atmosphere over the image. The atmospheric species (especially water vapor and ozone), the pressure, and the temperature profiles are set for the different altitudes of the atmospheric layers. For this purpose we have used models derived from radiosonde measurements.

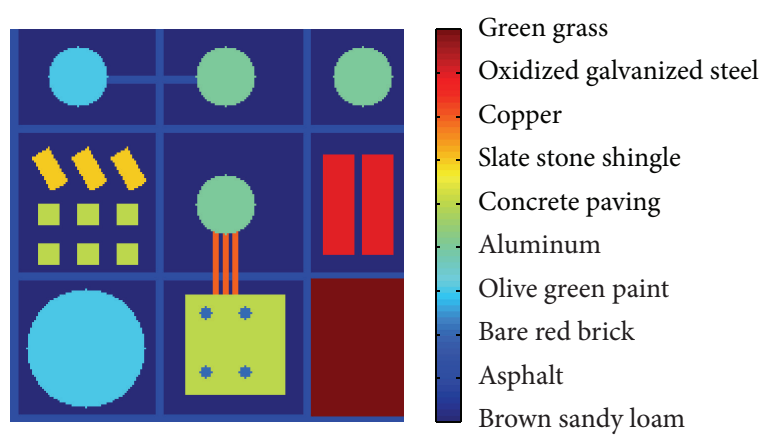

FIGURE 1: Spatial distribution of the different ground materials composing the scene.

At this stage, we generate a free-plume hyperspectral image of the scene, based on MODTRAN calculations, using COMANCHE software [24].

3.1.3. Gas Signature. The different gas species absorb light at various wavelengths. This phenomenon depends on the electronic, vibrational, and rotational bands of the gas molecules.

In this study, we selected two pollutant gases with very different radiative behavior. The sulfur dioxide $\left(\mathrm{SO}_{2}\right)$ has a spread absorption spectrum on the $7.5-12.5 \mu \mathrm{m}$ band as shown in Figure 4, while the ammonia $\left(\mathrm{NH}_{3}\right)$ presents several narrow spectral bands, especially for the wavelengths $10.42 \mu \mathrm{m}$ and $10.75 \mu \mathrm{m}$, which correspond to $960 \mathrm{~cm}^{-1}$ and $930 \mathrm{~cm}^{-1}$ in terms of wavenumber.

The absorption spectra of Figure 4 are taken from the high resolution library of the PNNL. These spectra were reduced to a $5 \mathrm{~cm}^{-1}$ spectral resolution to be consistent with typical sensor spectral resolution.

3.1.4. Plume Distribution Simulation. In order to introduce the plume's radiative impact to the free-plume signal, we have to model the $3 \mathrm{D}$ plume distribution in the scene, which is to define for each pixel of the plume the vertical profile of the present gases.

ADMS-Aircraft (Atmospheric Dispersion Modelling System) code [25] was used to generate a Gaussian plume based on the Brigg equation for plume dynamics [26]. The use of ADMS-Aircraft code requires the specification of some inputs related to the scene geometry, the atmospheric and meteorological conditions (in particular the wind speed and direction), and the plume initial conditions like the release rate/velocity/temperature.

Figure 5 shows the distribution of integrated concentration of a simulated $\mathrm{SO}_{2}$ plume with the following parameters:

(i) outflow rate $\left(\mathrm{SO}_{2}\right): 222 \mathrm{~g} / \mathrm{s}$,

(ii) outflow rate $\left(\mathrm{NH}_{3}\right): 11.39 \mathrm{~g} / \mathrm{s}$,

(iii) stack height: $20 \mathrm{~m}$,

(iv) stack radius: $3 \mathrm{~m}$,

(v) plume ejection speed: $2 \mathrm{~m} / \mathrm{s}$, 


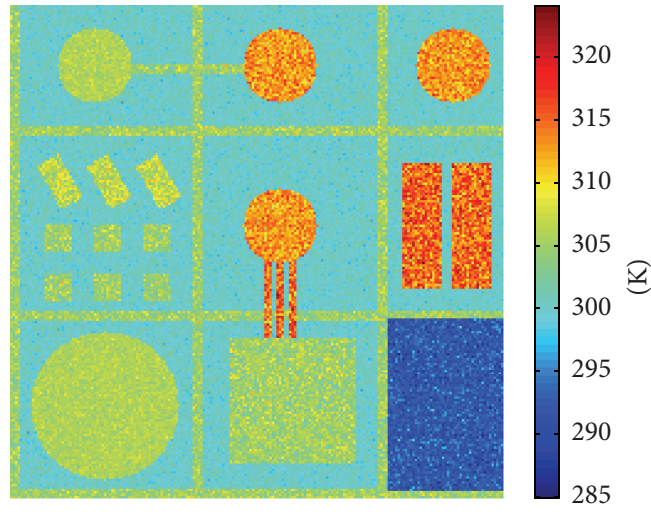

(a)

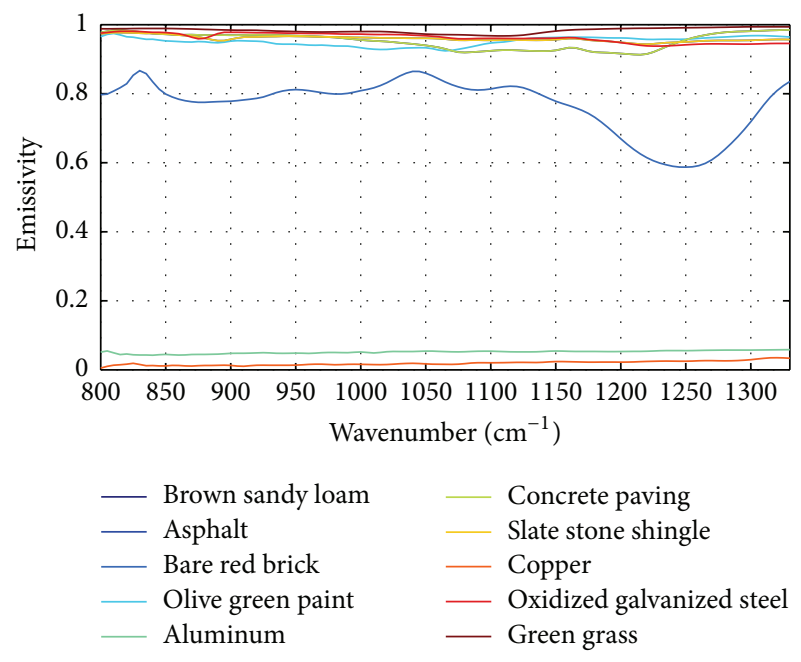

(b)

FIGURE 2: Ground parameters simulation. (a) Ground temperature map. (b) Emissivity spectra of materials present in the synthetic scene.

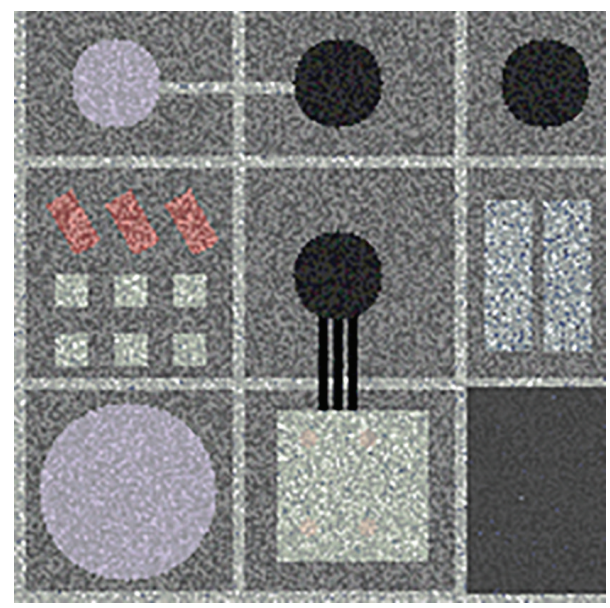

FIGURE 3: Synthetic refinery scene without plume. Image in RGB colors, representing the bands (R: $820 \mathrm{~cm}^{-1}, \mathrm{G}: 965 \mathrm{~cm}^{-1}$, and B: $\left.1165 \mathrm{~cm}^{-1}\right)$.

(vi) plume ejection direction: vertical,

(vii) wind speed: $2 \mathrm{~m} / \mathrm{s}$.

A plume temperature distribution simulation is also needed to introduce the plume's radiative impact on the free-plume signal. To introduce plume temperature distribution needed to estimate the plume's radiative impact on the free-plume signal, we only set the gas exit temperature assuming that $\Delta T$, the difference between the plume temperature $\left(T_{p}\right)$ and the initial ambient temperature $\left(T_{\mathrm{a}}\right)$, follows the same distribution compared to the gas concentration [11]. In our simulation we fixed $\Delta T$ at the emission source to $300 \mathrm{~K}$.

The radiance signal of the plume-present scene is represented in Figure 6 and that of the plume-absent scene in Figure 3. This signal is obtained using COMANCHE software that takes into account all the parameters described above.

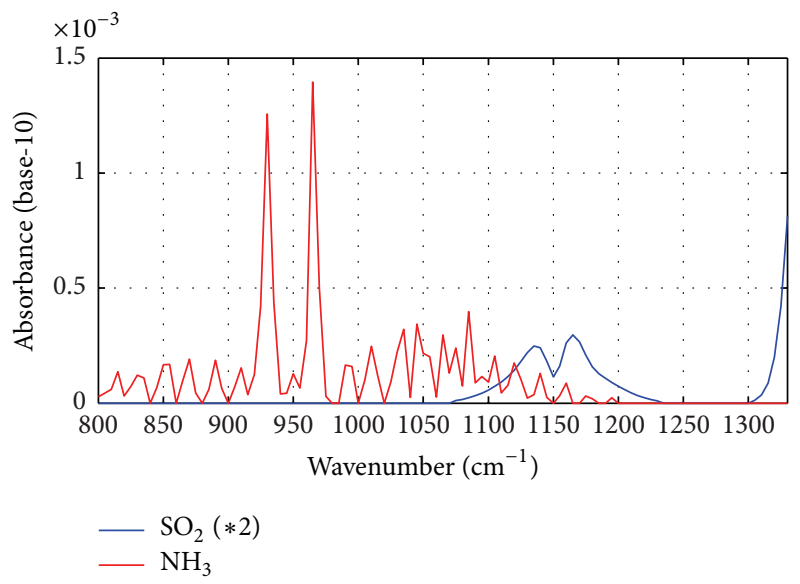

Figure 4: Absorptive coefficient spectra of sulfur dioxide (blue curve) and ammonia (red curve).

\subsection{Results and Discussion}

3.2.1. Plume Detection. The first step performed is the plume detection. The SMF algorithm was applied twice: the first time for detecting $\mathrm{SO}_{2}$. The absorbance of this gas was used to model the associated target vector $\mathbf{T}$. The second time the vector $\mathbf{T}$ was taken equal to the absorbance of $\mathrm{NH}_{3}$. After two thresholding operations on these results, we consider that the plume region is the union of the two masks. Note that a morphological opening operation was performed in order to remove the detection artifacts.

Figure 7 represents the plume mask obtained at the end of these treatments.

3.2.2. Classification of the Synthetic Scene. In this subsection, we evaluate the efficiency of the proposed classification method. For this purpose, the confusion matrix and the Cohen kappa coefficient are used. 


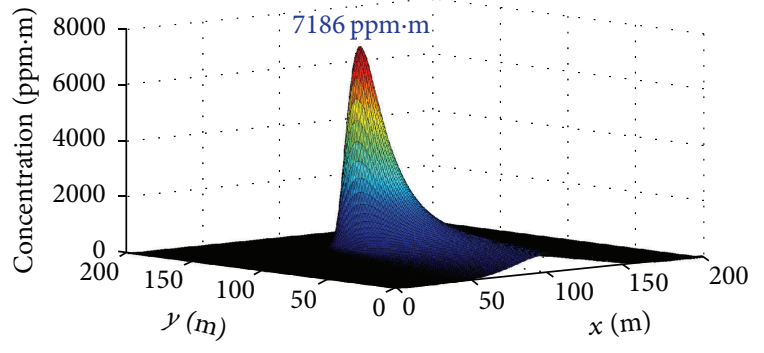

FIGURE 5: Distribution of $\mathrm{SO}_{2}$ concentration of the simulated plume, with an outflow rate of $222 \mathrm{~g} / \mathrm{s}$ and a wind speed of $2 \mathrm{~m} / \mathrm{s}$.

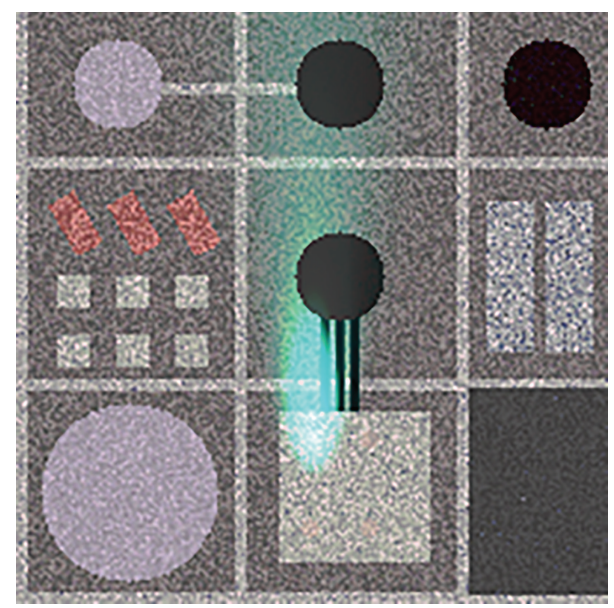

FIGURE 6: Synthetic refinery scene with a Gaussian plume of $\mathrm{SO}_{2}$ and $\mathrm{NH}_{3}$. Image in RGB colors, representing the bands (R: $820 \mathrm{~cm}^{-1}, \mathrm{G}$ : $965 \mathrm{~cm}^{-1}$, and B: $1165 \mathrm{~cm}^{-1}$ ).

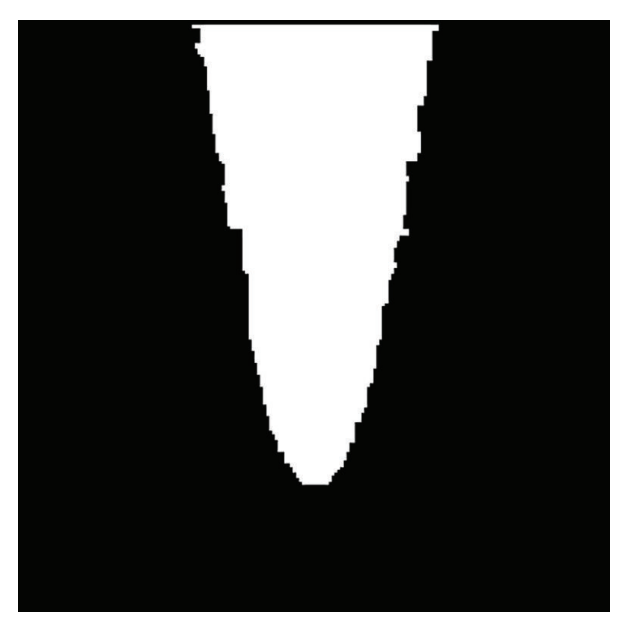

Figure 7: Plume detection mask for the synthetic refinery scene.

In Figure 8, we illustrated the classification results on the synthetic refinery scene, both for the plume-absent area (Figure 8(a)) and the plume-present area (Figure 8(b)).

Otherwise, in Table 1, we figured the confusion matrix of the two classifications realized. This matrix represents, for each material present in the synthetic scene, its distribution
TABLE 1: Confusion matrix of the classifications performed on the synthetic refinery scene.

(a)

\begin{tabular}{lccccc}
\hline \multirow{2}{*}{ Material } & \multicolumn{5}{c}{ Classes of the plume-absent area } \\
& 1 & 2 & 3 & 4 & 5 \\
\hline Green grass & 3149 & 141 & 0 & 0 & 0 \\
Brown sandy loam & 3 & 15241 & 121 & 0 & 0 \\
Olive green paint & 0 & 15 & 3165 & 350 & 0 \\
Asphalt & 0 & 410 & 2543 & 999 & 0 \\
Bare red brick & 0 & 0 & 51 & 36 & 0 \\
Concrete paving & 0 & 206 & 1695 & 891 & 0 \\
Slate stone shingle & 0 & 380 & 220 & 3 & 0 \\
Oxidized & 14 & 686 & 734 & 198 & 0 \\
galvanized steel & 0 & 0 & 0 & 0 & 709 \\
Aluminum & & & &
\end{tabular}

(b)

\begin{tabular}{lcccc}
\hline \multirow{2}{*}{ Material } & \multicolumn{5}{c}{ Classes of the plume-present area } \\
& 1 & 2 & 3 & 4 \\
\hline Brown sandy loam & 5145 & 9 & 0 & 0 \\
Asphalt & 128 & 612 & 0 & 0 \\
Bare red brick & 0 & 29 & 0 & 0 \\
Concrete paving & 46 & 373 & 0 & 0 \\
Copper & 0 & 0 & 60 & 220 \\
Aluminum & 0 & 0 & 0 & 1418 \\
\hline
\end{tabular}

according to the obtained clusters. The classification of the plume-absent region yields to 5 clusters, while the plumepresent classification leads to 4 clusters.

From Figure 8 and Table 1, one can notice that materials could be sorted into three different families: Green grass, \{Aluminum + Copper\}, and the other materials of the scene. This result is consistent with the ground parameters distribution: the surface temperature of the Green grass is lower than the temperature of other materials (see Figure 2(a)). Moreover, aluminum and copper are reflective materials, unlike the other materials (see Figure 2(b)).

Inside the two last families of materials, the discrimination is quite difficult to achieve. Indeed, the radiances of those materials are very close. Nevertheless, the performed classifications are rather successful. The Cohen kappa coefficient is equal to 0.61 for the plume-absent classification; the latter equals 0.81 for the plume-present classification. According to the characterization done by Landis and Koch [27] on the values of the kappa coefficient, our classifications present a substantial (0.61) agreement and an almost perfect agreement (0.81).

3.2.3. Comparison of Background Radiance Estimation Methods. In order to compare the performance of the three methods used to estimate the background radiance, four pixels through the plume were picked out. These pixels, belonging 


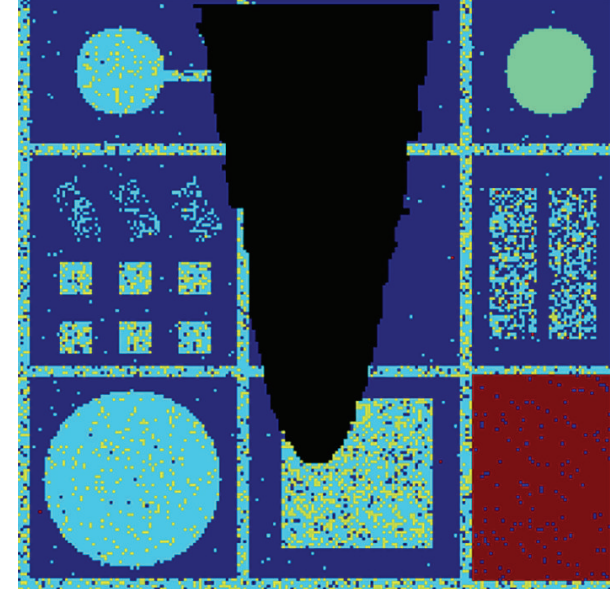

(a)

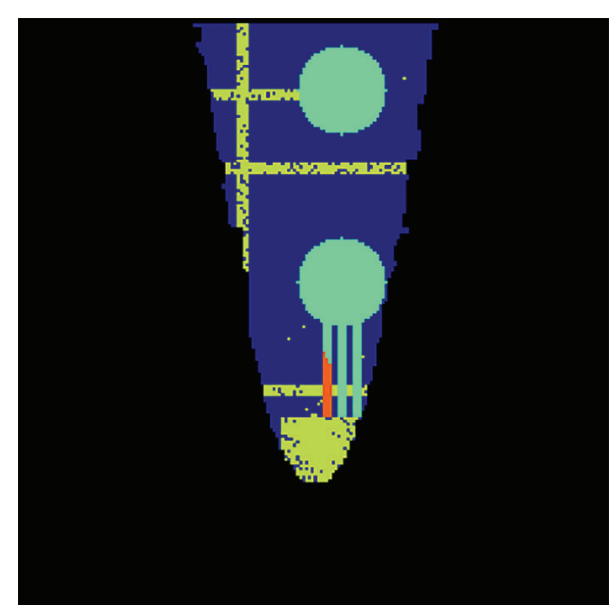

(b)

Figure 8: Classification results on the synthetic refinery scene. (a) Plume-absent region. (b) Plume-present region.

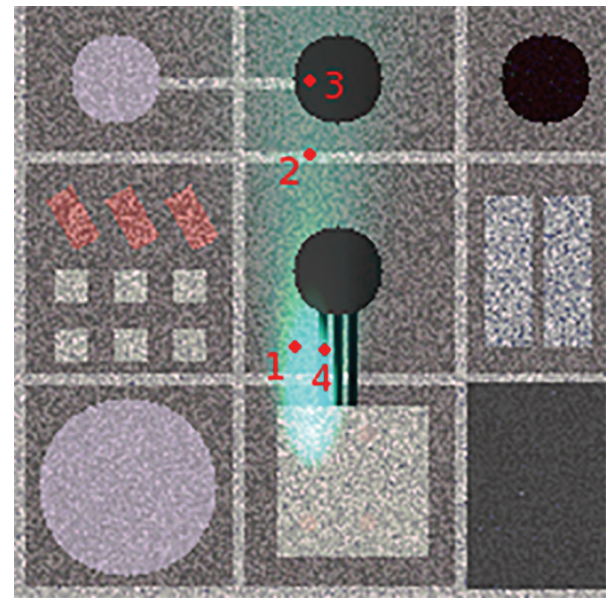

Figure 9: Positions of used pixels in the background radiance estimation methods comparison.

to different classes derived from the classification (see Section 3.2.2), are figured hereafter in Figure 9. The ground of these pixels is composed, respectively, of brown sandy loam, asphalt, aluminum, and copper.

Figure 10 summarizes the results of background radiance estimation using the three methods: SB, CB, and CSB. For each of the selected pixels are represented the on-plume radiance (red), the real off-plume radiance (dotted green), and the estimation of $L_{\mathrm{bkg}}$ using SB (cyan), CB (blue), and CSB (magenta).

The cluster of brown sandy loam is the most present among plume-absent pixels. Thus, the background properties of the latter are strongly represented in the principal vectors derived from the PCA. It is therefore obvious that the SB method gives an accurate estimate of the background radiance for pixels of this cluster (Figure 10(a)).

Furthermore, the background radiances of asphalt cluster pixels are pretty close to the background radiances of brown sandy loam cluster pixels. That is why $L_{\mathrm{bkg}}$ estimate given by $S B$ approach remains rather effective for this cluster (Figure 10(b)).

Nonetheless, reflective materials clusters have a background radiance much far from the main plume-absent cluster, which affects significantly the accuracy of the background radiance estimation using SB, as we notice in Figures 10(c) and $10(\mathrm{~d})$.

We observe in these two last figures that, for such materials, SB method reproduces approximately the high frequency variations. However, there is a component similar to the main cluster background radiance, which adds up to the estimation.

These curves indicate at one hand that the SelectedBand approach yields an accurate estimate of the background radiance, for the pixels of the most represented class among plume-absent pixels. But on the other hand, for other classes, the less the class is represented outside the plume, the less its background radiance is correctly estimated. From these observations, we deduce that SB method does not manage well the case of heterogeneous background estimation.

Figure 10 shows that the introduction of a classification to the background radiance estimation process improves the results, especially for the minority classes of the scene. For those pixels (Figures 10(c) and 10(d)), the two classificationbased methods (CB and CSB) give a more accurate estimation than SB does.

One can note that, for pixel 4 , there is a slight difference between the real $L_{\mathrm{bkg}}$ radiance and the estimated one using $\mathrm{CB}$ or CSB. This difference comes from the fact that the class \#4 is not represented at all in the plume-absent region. Thus, it is assimilated during the classification with the class \#3.

Regarding to the pixels 1 and 2, the results of the method $\mathrm{CB}$ are less specific than those of the SB method. Indeed, $\mathrm{CB}$ method does not take into account the intraclass variability by assimilating to each plume-present pixel the mean radiance of plume-absent pixels belonging to the same class of the latter. To this limitation, we must add the errors due to 


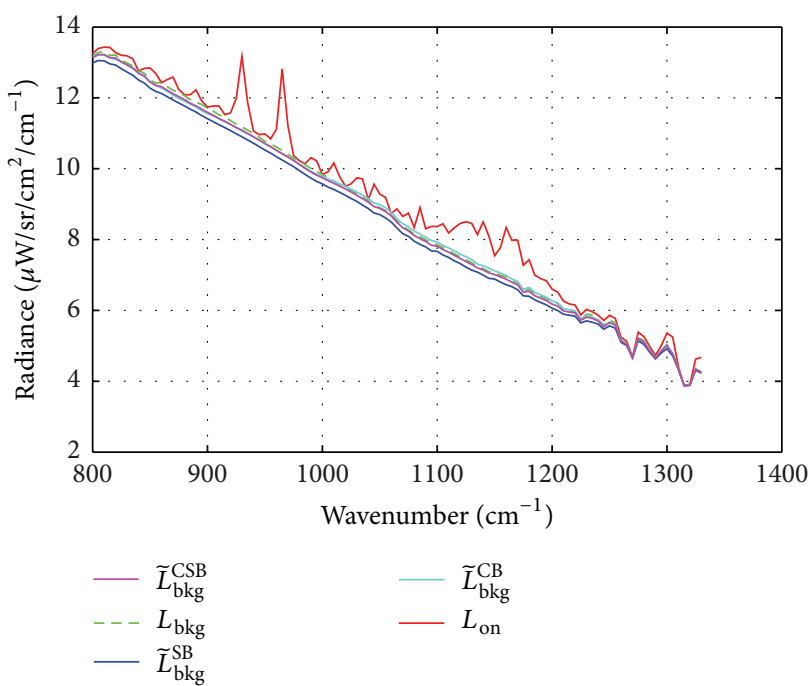

(a)

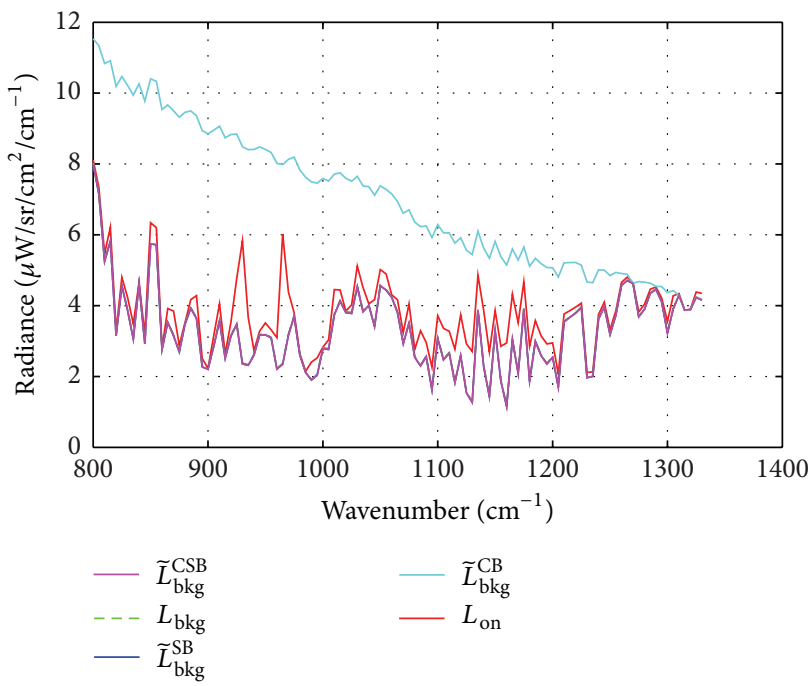

(c)

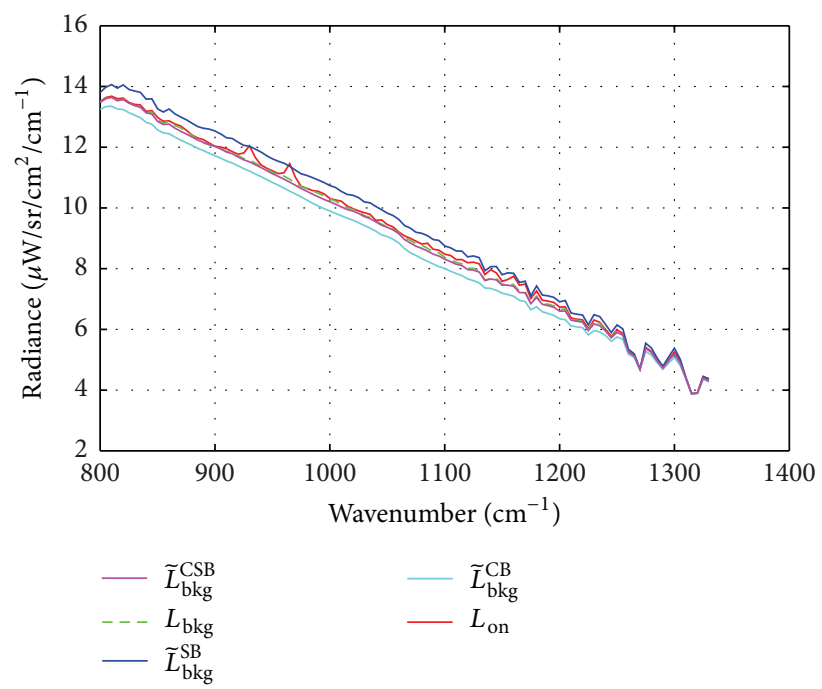

(b)

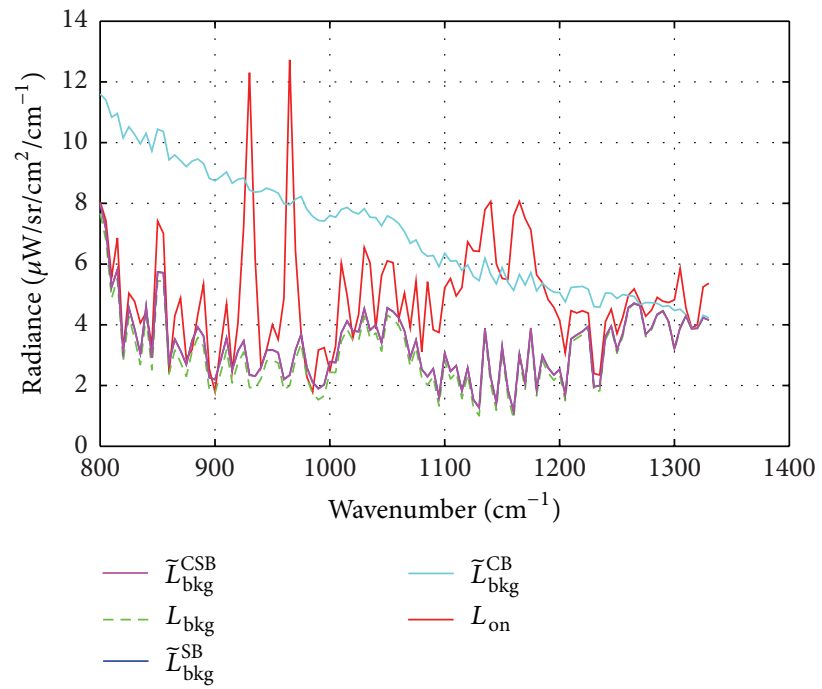

(d)

FIGURE 10: Comparison of background radiance estimation methods, for four different pixels through the plume. The red curves stand for the on-plume radiances; the dotted green ones are the real background radiances. The cyan, blue, and magenta curves represent, respectively, the background radiance estimated by the SB, CB, and CSB methods. (a) Results for pixel 1 (brown sandy loam), (b) results for pixel 2 (asphalt), (c) results for pixel 3 (aluminum), and (d) results for pixel 4 (copper).

the missclassification. Despite these two sources of errors, the CB background radiance estimation is still acceptable.

Concerning the CSB method, the estimation coincides perfectly with the real background radiance, for those pixels. This method manages well the intraclass variability.

In order to have a spatial visualization of the estimation error of the three compared methods, we illustrated in Figure 11 the mean error of background radiance estimation. This mean error was expressed in terms of brightness temperature.

Figure 11 upholds the undermentioned results. As expected, with the SB approach, we obtain a mean error less than $5 \mathrm{~K}$ for the classes which are the most represented among plume-absent pixels (\#1 and \#2). This result confirms the efficiency of the approach on homogeneous scene. However, for the two other classes, which are poorly (\#3) or not (\#4) represented among the plume-absent pixels, we reach more than $45 \mathrm{~K}$ of error.

In the case of Clustering-Based methods, the mean error obtained for the minority clusters is far smaller than the error gotten with SB approach. Through class \#4 the mean error is less than $4.5 \mathrm{~K}$, both for CB and CSB methods, despite the fact that the latter is not represented among the plume-absent pixels.

For the other classes, even class \#3 which presents more than $35 \mathrm{~K}$ of error with the SB approach, we hardly reach less than $2.5 \mathrm{~K}$ of error (CSB method). With the $\mathrm{CB}$ method, some pixels have a mean error reaching $5.5 \mathrm{~K}$, but those pixels are very few. 


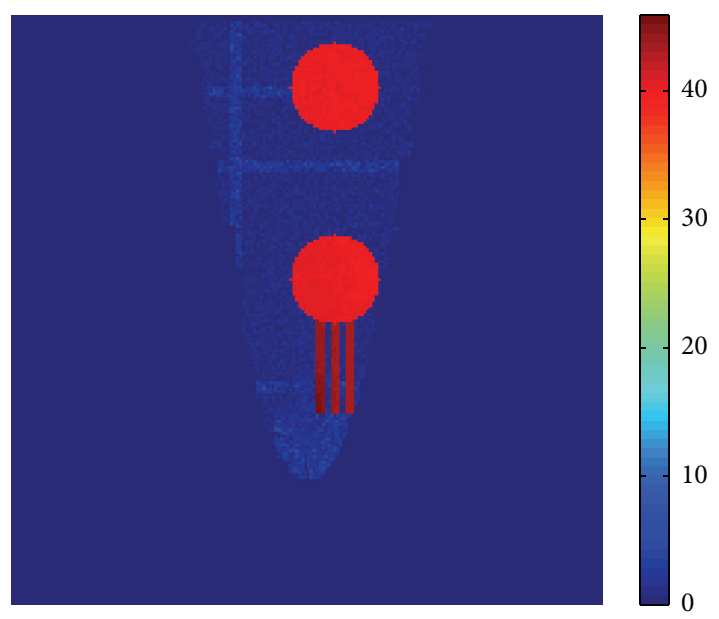

(a)

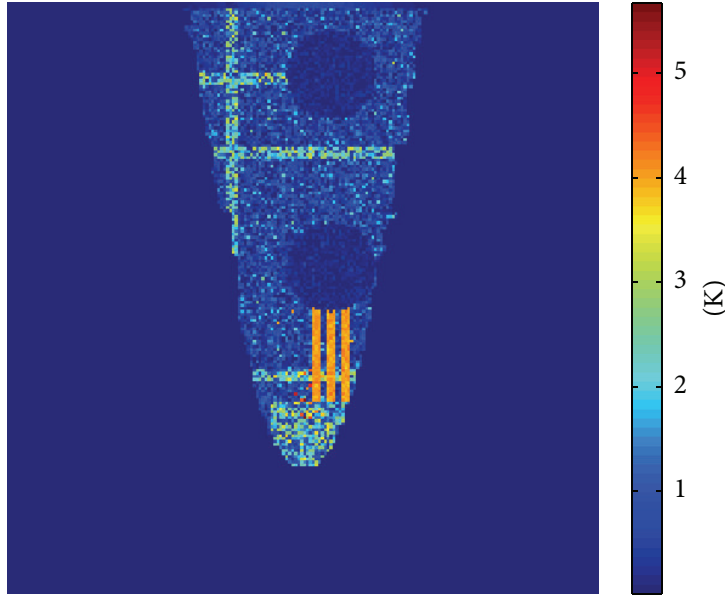

(b)

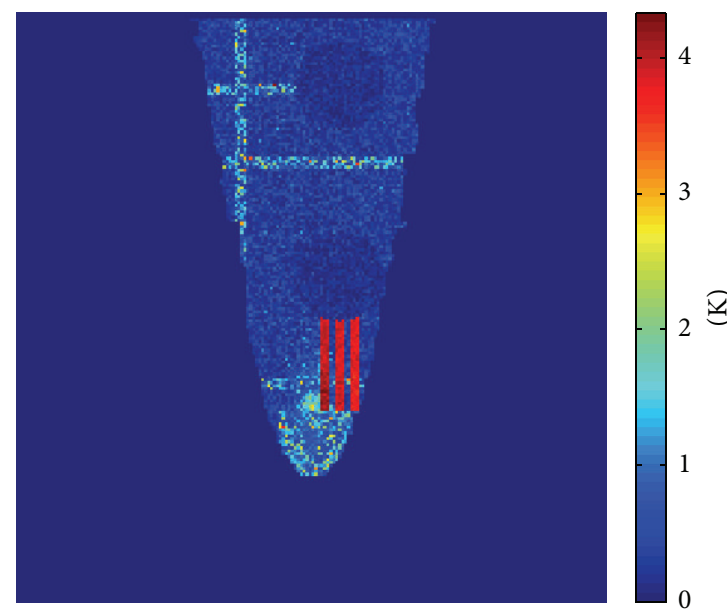

(c)

FIGURE 11: Mean error of background radiance estimation, expressed in terms of brightness temperature. (a) Mean error obtained with the Selected-Band estimation method. (b) Mean error obtained with the Clustering-Based estimation method. (c) Mean error obtained with the Clustering-Based Selected-Band estimation method.

To assess the overall performance of these three methods, the average error was calculated over the plume. As expected, the CSB method is the best of the three methods: the mean error obtained over the plume is equal to $0.48 \mathrm{~K}$. With the $\mathrm{CB}$ method, the mean error remains low; it equals $0.80 \mathrm{~K}$. However, the SB method gives a mean error of $9.55 \mathrm{~K}$ over the plume. This is explained by the large error of estimation for classes \#3 and \#4.

After carrying out the three methods on synthetic data, we can conclude that CSB approach provides better results than $\mathrm{CB}$ and $\mathrm{SB}$ approaches, particularly regarding materials not well, or not at all, represented among the plume-absent pixels.

\section{Application on Real Data}

4.1. Presentation of Telops Hyper-Cam Acquisition. The Telops Hyper-Cam LW is a lightweight and compact imaging instrument which uses Fourier Transfer Infrared (FTIR) technology. The spectral resolution is user-selectable up to $0.25 \mathrm{~cm}^{-1}$ over the 7.7 to $11.7 \mu \mathrm{m}$ spectral range. The groundbased Telops Hyper-Cam is installed on a stabilization platform equipped with a global positioning system (GPS) and inertial motion unit (IMU). In a FTS imaging system, signal modulation is achieved using a Michelson interferometer. Acquiring a full interferogram typically lasts about one second. Therefore, an image motion compensation mirror uses GPS/IMU data to compensate efficiently for the aircraft movements during data acquisition.

This flight was carried out using a Hyper-Cam LW sensor at an altitude of 685 meters and a speed of 110 knots leading to a ground pixel size of $0.057 \mathrm{~m}^{2} /$ pixel. A spectral resolution of $6 \mathrm{~cm}^{-1}$ was used which gives a total of 90 spectral bands equally spaced over the whole range cover by the focal pane array detector. Outside temperature, wind speed, and relative humidity at ground level were $21^{\circ} \mathrm{C}, 15 \mathrm{~km} / \mathrm{h}$, and $37 \%$, respectively. 


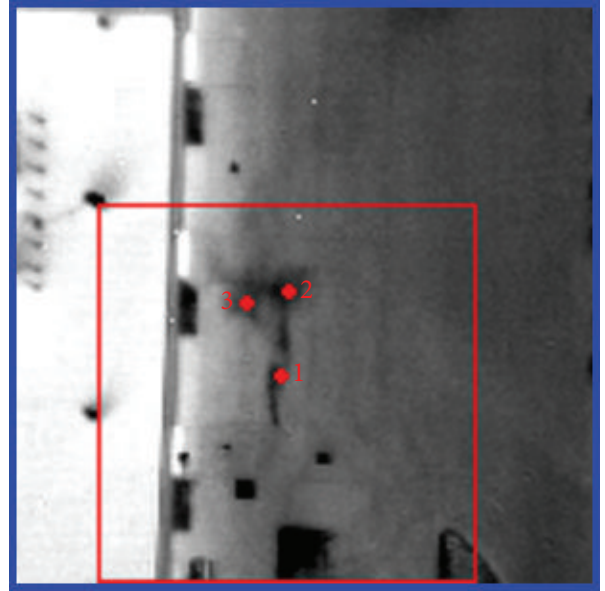

(a)

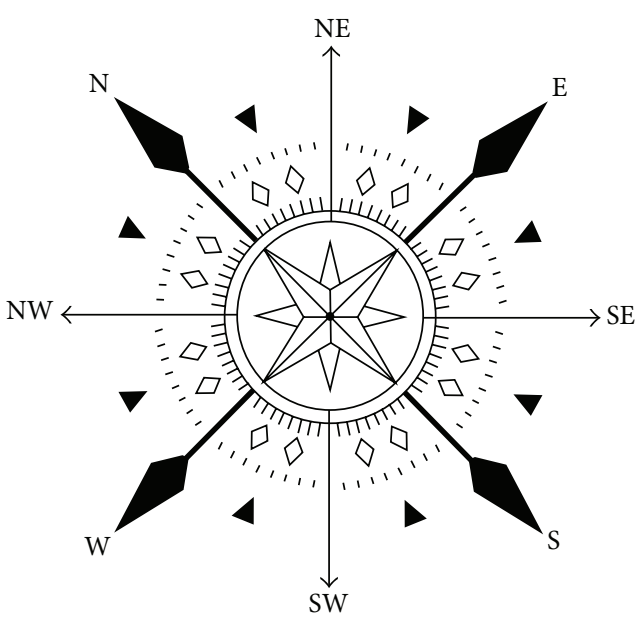

(b)

FIGURE 12: Illustration of subimage and pixels used in the background radiance estimation methods comparison.

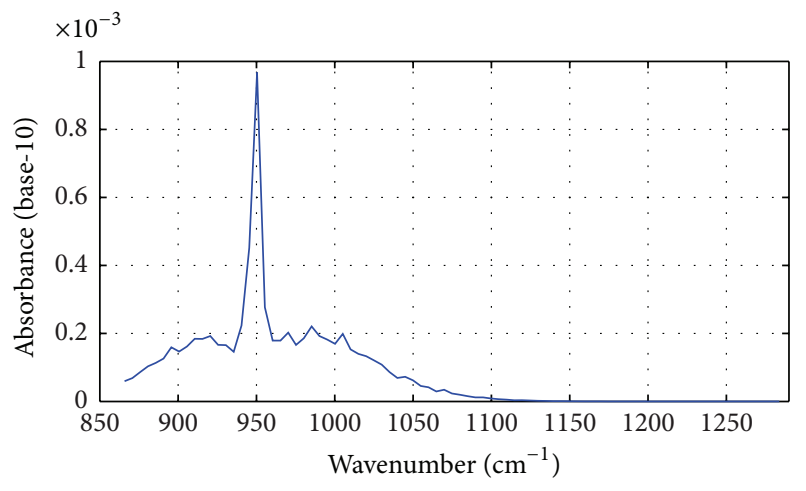

(a)

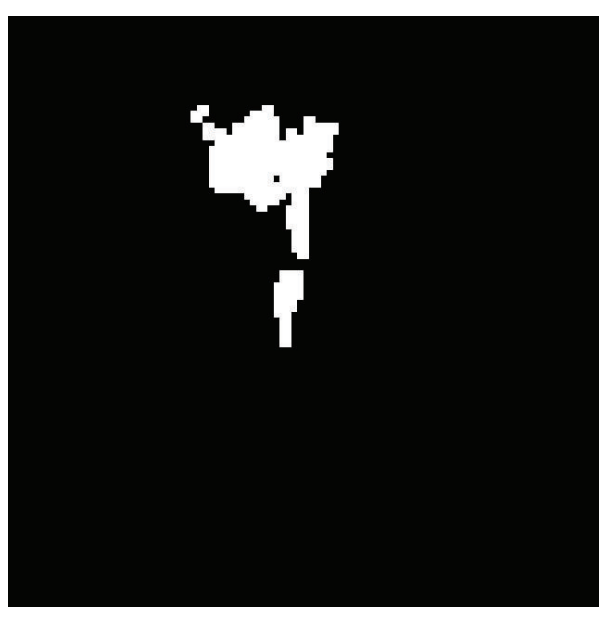

(b)

FIGURE 13: Ethylene release detection. (a) Absorptive coefficient spectrum of ethylene (from PNNL). (b) Plume detection mask for Telops parking scene.

Airborne hyperspectral infrared measurements were carried out above a pure ethylene gas released. Ethylene is a flammable gas massively used worldwide in the production of many polymer materials. During this experiment, gas was released at a constant flow rate of approximately $20 \mathrm{~L} / \mathrm{min}$.

\subsection{Ground Radiance Estimation. Since the ethylene plume} covers a ground composed entirely of asphalt, we reduced the size of the image to be processed, in order to limit the number of classes obtained in the plume-absent area. This $100 \times 100$ subimage is represented by the red square in Figure 12.

4.2.1. Ethylene Presence Detection. The SMF algorithm was applied on the Telops acquisition in order to define the ethylene release spatial extension. The ethylene's absorbance curve, illustrated in Figure 13(a), was used to model the target vector $\mathbf{T}$ (see Section 2.1).

A plume mask (Figure 13(b)) is obtained after the application of a threshold on the abundances image $\boldsymbol{\alpha}$. In addition, we performed a morphological opening operation, in order to take away the detection artifacts.

Unfortunately the applied morphological opening removes some plume-present pixels, which are localized at the frontier of the plume.

4.2.2. Classification of Telops parking Scene. As opposed to the synthetic scene case, we cannot have a quantitative evaluation of the classification. Thus, only a qualitative description of it is given thereafter.

On this acquisition performed around 11 a.m., one can notice the presence of a temperature gradient along the 


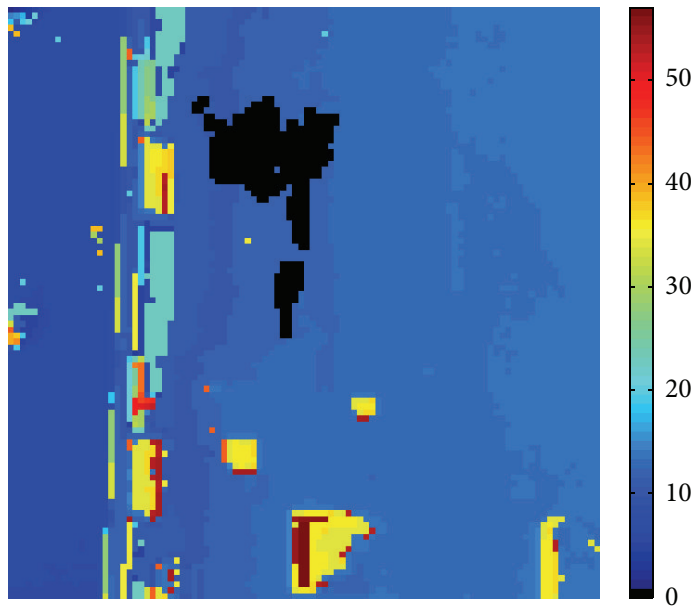

(a)

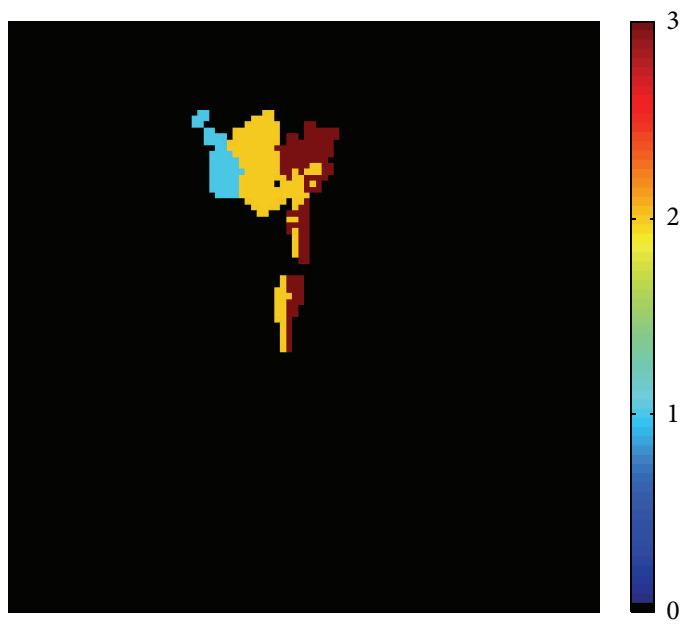

(b)

FIgure 14: Classification results on Telops parking scene. (a) Plume-absent region. (b) Plume-present region.

southeast to northwest direction. Indeed, the sun, while rising in the sky, heated gradually the scene. Thus, even if two pixels are composed from the same material, they could have two different ground temperature, according to their location in the scene. Consequently, those pixels should be classified into two different clusters.

Figure 14 shows the classification results obtained on Telops' parking scene. The classification on the plume-absent region is illustrated in Figure 14(a), while the classification involving the plume-present region is illustrated in Figure $14(\mathrm{~b})$.

The plume-present region was clustered into 3 classes, while the plume-absent region was clustered into 57 classes.

From these two figures, we notice that the classification complies with the temperature gradient. Note however that the classification in the plume-present region is more accurate than the second classification. Indeed, the plumeabsent classes corresponding to the plume-present classes \#2 (yellow) and \#3 (brown) were merged into the same class. This can be explained by the fact that we used a threshold on the size of class $\left(D_{\max }\right)$ higher in the plume-absent case, in order to reduce the number of classes.

\subsubsection{Comparison of Background Radiance Estimation Meth-} ods. For this scene, we do not have any a priori knowledge of the background radiance. But, by observing the scene, we can notice that it seems to be homogeneous along the vertical direction. In other words, it is justified to assume that $L_{\mathrm{bkg}}$ is vertically constant. Hence, for a given plume-present pixel, we approximate its background radiance by the mean spectra of some plume-absent pixels located at the same column than the latter. This radiance will be designated by the term $\left\langle L_{\text {off }}\right\rangle$.

Figure 15 shows the results of background radiance estimation using the three methods: SB, CB, and CSB. For the three selected pixels, we figured the on-plume measured radiances (red curve), the expected off-plume radiances (dotted green curve), and the estimation of $L_{\mathrm{bkg}}$ using $\mathrm{SB}$ (cyan), CB (blue), and CSB (magenta).
The first point we can deduce from Figure 15 is the relevance of the radiance $\left\langle L_{\text {off }}\right\rangle$ to model the background radiance. One can notice that it agrees quite well with the onplume radiance on bands where the absorbance of ethylene is close to zero. Moreover, the difference between $\left\langle L_{\text {off }}\right\rangle$ and $L_{\text {on }}$ on bands of ethylene absorption follows the same variation then the absorption curve of ethylene.

For the three chosen pixels, the background radiance estimation obtained by both CB and CSB methods is rather consistent with $\left\langle L_{\text {off }}\right\rangle$. Nonetheless, the SB estimate does not match well with the expected radiance. For pixels \#1 and \#2 SB approach underestimates the background radiance, while it overestimates $L_{\mathrm{bkg}}$ for pixel \#3.

Those estimation errors are distributed over the whole spectral range. They are more important for ethylene absorption features.

So as to get an idea of the error magnitude for each method, two parameters measuring this error with respect to the expected radiance were defined: the root mean square of the brightness temperature error $\left(\sigma_{T_{\mathrm{b}}}\right)$ is calculated, for each pixel, as follows:

$$
\sigma_{T_{\mathrm{b}}}(\mathrm{K})=\sqrt{\frac{1}{N_{\mathrm{B}}} \cdot\left(\widetilde{T_{\mathrm{bkg}}}-\left\langle T_{\mathrm{off}}\right\rangle\right) \cdot\left(\widetilde{T_{\mathrm{bkg}}}-\left\langle T_{\mathrm{off}}\right\rangle\right)^{T}}
$$

where $\widetilde{T_{\mathrm{bkg}}}$ is the background brightness temperature estimated by one of the three methods, for the concerned pixel. $\left\langle T_{\text {off }}\right\rangle$ is the expected background brightness temperature, for pixel in question.

Moreover, we define the relative root mean square error on radiance $\left(\sigma_{L}\right)$ by

$$
\begin{aligned}
& \sigma_{L}(\%) \\
& \quad=\sqrt{\frac{1}{N_{\mathrm{B}}} \cdot\left(\frac{\widetilde{L_{\mathrm{bkg}}}-\left\langle L_{\mathrm{off}}\right\rangle}{\left\langle L_{\mathrm{off}}\right\rangle}\right) \cdot\left(\frac{\widetilde{L_{\mathrm{bkg}}}-\left\langle L_{\mathrm{off}}\right\rangle}{\left\langle L_{\mathrm{off}}\right\rangle}\right)^{T}},
\end{aligned}
$$




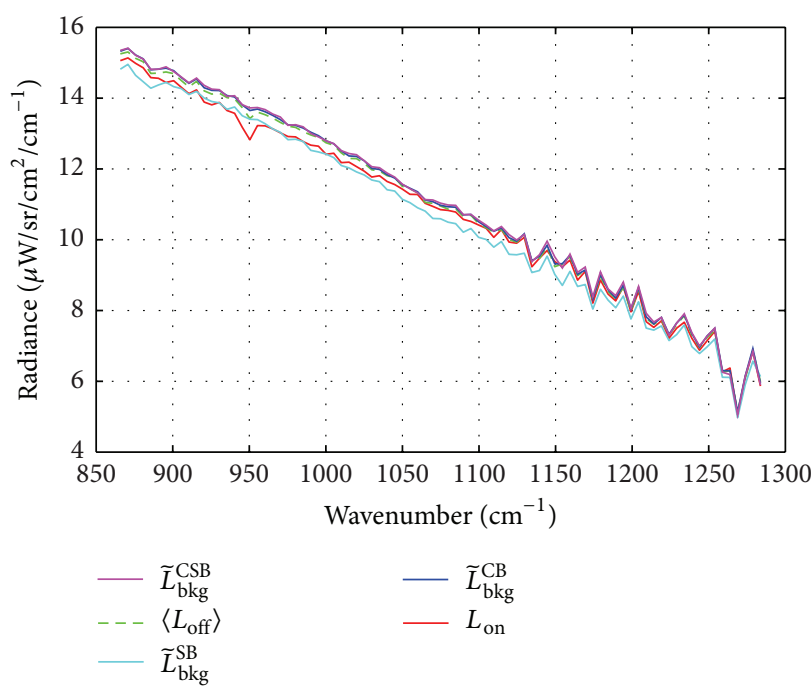

(a)

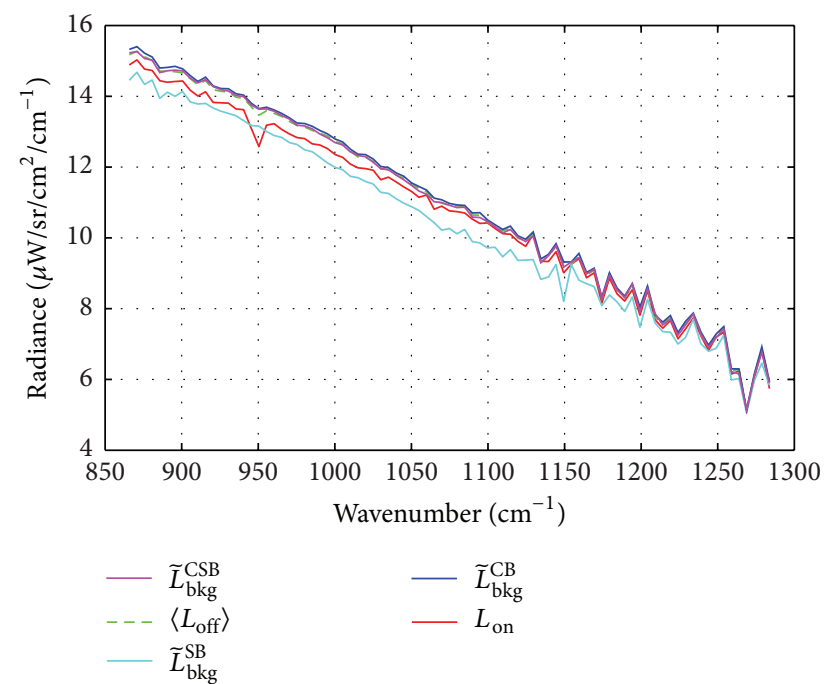

(b)

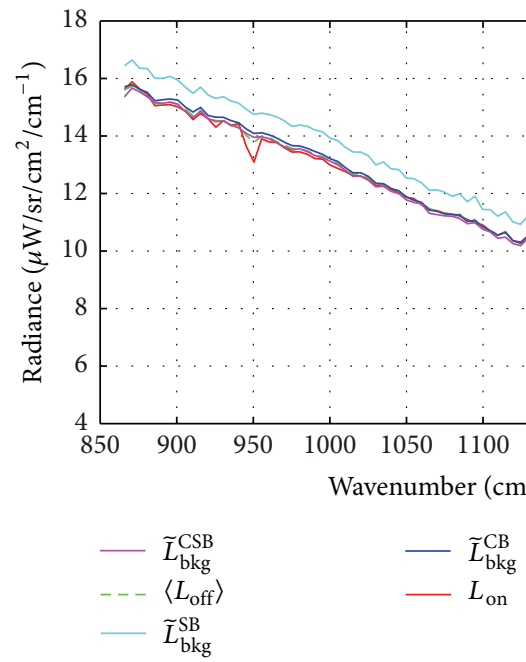

(c)

FIGURE 15: Comparison of background radiance estimation methods, for three different pixels through the plume. The red curves stand for the on-plume radiances; the dotted green ones are the expected background radiances. The cyan, blue, and magenta curves represent, respectively, the background radiance estimated by the SB, CB, and CSB methods. (a) Results for pixel 1, (b) results for pixel 2, and (c) results for pixel 3.

where $\widetilde{L_{\mathrm{bkg}}}$ is the background radiance estimated by one of the three methods, for the concerned pixel.

We figured the obtained results, for each of the three pixels, in Table 2.

Table 2 validates the conclusions deduced from Figure 15. The SB method gives a less accurate estimation than the Clustering-Based methods. If for pixel \#1, the mean error calculated is three times higher with the SB method, it becomes ten times higher for pixels \#2 and \#3. Otherwise, the $\mathrm{CB}$ method has a background radiance estimation almost as similar as the CSB method estimation. Beside for pixel \#1, the estimation obtained by the $\mathrm{CB}$ method is better than that obtained by CSB method. Moreover, the average error of all plume-present pixels is equal to $0.64 \mathrm{~K}$ for the $\mathrm{CB}$ method, while it is equal to $0.68 \mathrm{~K}$ for the CSB method. However the two methods remain of the same order of magnitude.
Recall, nevertheless, that $\left\langle L_{\text {off }}\right\rangle$ is just an approximation of the real $L_{\text {off }}$. Thus, the result presented below does not induce necessarily that $\mathrm{CB}$ method is better than CSB method.

Figure 16 illustrates a comparison of different radiances at the ethylene most absorptive band $\left(950.4 \mathrm{~cm}^{-1}\right)$, for pixels along a horizontal cross section. Pixels from 35 to 55 are plume-present pixels. Therefore, the radiances comparison is restricted to those pixels.

Figure 16 shows that the SB approach miscalculates the background radiance, comparing to the other methods. On a part of plume-present pixels, it overestimates $L_{\mathrm{bkg}}$, while it underestimates this term on the other parts of plume-present pixels. The estimation error reaches at most $10 \%$ for this band. Recall that this band is the most important for the ethylene quantification (see Figure 13(a)). 
TABLE 2: Evaluation of the difference between expected background radiance and estimated background radiance, using one of the three methods used in this paper. The first table presents the root mean square of the brightness temperature error $\left(\sigma_{T_{\mathrm{b}}}\right)$, while the second summarizes the results in terms of the relative root mean square error on radiance $\left(\sigma_{L}\right)$.

(a)

\begin{tabular}{lccc}
\hline$\sigma_{T_{\mathrm{b}}}(\mathrm{K})$ & \multicolumn{3}{c}{ Selected pixels } \\
\hline $\mathrm{SB}$ & 1 & 2 & 3 \\
$\mathrm{CB}$ & 1.96 & 3.32 & 3.94 \\
$\mathrm{CSB}$ & 0.36 & 0.44 & 0.46 \\
\hline
\end{tabular}

(b)

\begin{tabular}{lccc}
\hline$\sigma_{L}(\%)$ & \multicolumn{3}{c}{ Selected pixels } \\
& 1 & 2 & 3 \\
\hline $\mathrm{SB}$ & 3.1 & 5.06 & 6.21 \\
$\mathrm{CB}$ & 0.55 & 0.69 & 0.68 \\
$\mathrm{CSB}$ & 0.95 & 0.5 & 0.67 \\
\hline
\end{tabular}

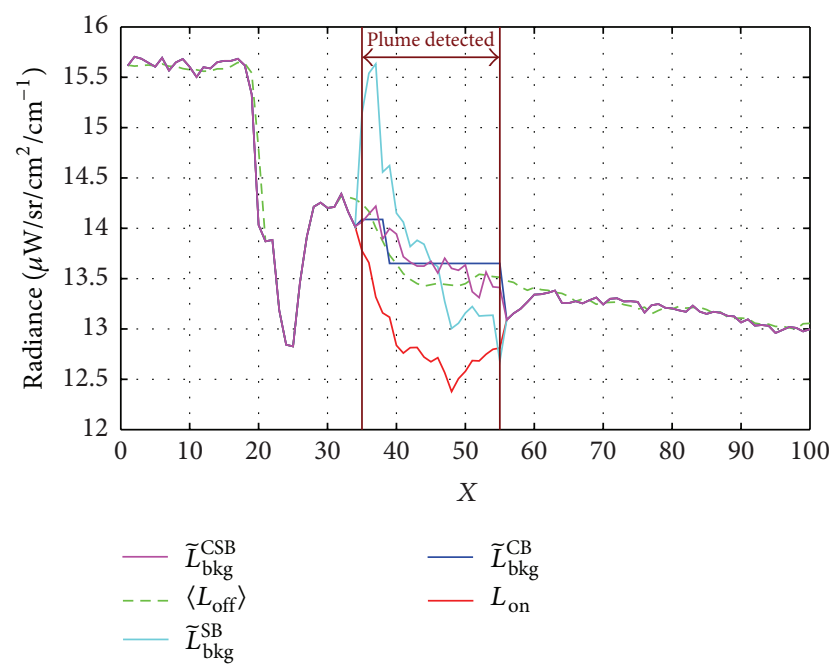

FIGURE 16: Comparison of different radiances at the ethylene absorptive band $\left(950.4 \mathrm{~cm}^{-1}\right)$, along a horizontal cross section containing some plume-present pixels. The red curve stands for the on-plume radiances; the green dotted one is the expected background radiances. The cyan, blue, and magenta curves represent, respectively, the background radiances estimated by the $\mathrm{SB}, \mathrm{CB}$, and CSB methods.

As expected, the radiance estimated using $\mathrm{CB}$ method is a constant piecewise function. We can see that, for the first class (from the left), the estimated radiance is consistent with the mean of the expected radiance $\left(\left\langle L_{\text {off }}\right\rangle\right)$ of concerned pixels. But for the second class, which is the result of merging of two classes (see Figure 14), the estimated radiance does not match perfectly with the mean of $\left\langle L_{\text {off }}\right\rangle$. Using a better classification of the plume-absent region, it is possible to sort this second class into two classes, which improves the estimation yielded by $\mathrm{CB}$ method.
In Figure 16, we observe that CSB method takes into consideration the intraclass variability, which allows getting better estimation with this method than CB method. For the concerned band, CSB method does not exceed 3\% of estimation error.

In order to generalize those observations to the all plumepresent pixels, we illustrated in Figure 17 a comparison of the background radiance estimation obtained by each of the three methods. This comparison is performed at the band $950.4 \mathrm{~cm}^{-1}$.

One can notice that at the frontier of the plume, some plume-present pixels have a relatively low radiance (dark pixels). This is due to a bad plume detection in this region as we already explained it above. For those pixels, the radiance represented in Figure 17 is $L_{\text {on }}$ and not $L_{\mathrm{bkg}}$.

Figure 17(a) shows that SB method overestimates the background radiance on a part of plume-present pixels (bright area of the plume). On another part of plume-present pixels, $L_{\mathrm{bkg}}$ is underestimated (dark area of the plume). This is due to the presence in the plume-absent region of more or less shinny materials than the plume-present pixels. The signal of those materials is then integrated in the principal components derived from the PCA.

The Clustering-Based methods use only the signal of plume-absent pixels having the same background properties than the plume-present pixels. The use of only pertinent information for the background radiance estimation therefore allows improving its accuracy.

This is clearly visible in Figure 17, since we note a certain homogeneity between the value of the plume-present and the plume-absent radiance.

4.3. Gas Concentration Estimation. From the scene described in Figure 12, we first compute an atmospheric compensation; then we apply a linear algorithm to estimate ethylene concentration for each background estimation.

Atmospheric compensation stage has two goals: first it aims to avoid the introduction of a probable bias from atmospheric nontarget gases on the retrieval; then it allows the estimation of the atmospheric ground level temperature and water vapor concentration. We assume here that atmosphere properties are homogeneous over the scene. We applied a Spectral Smoothness (SpSm) algorithm [28, 29] on a homogeneous subimage to estimate atmospheric profiles (temperature, water vapor), surface emissivity, and surface temperature.

The SpSm algorithm from a set of initial guess is used to reduce retrieved emissivity spectral variability on homogeneous area of concrete material. The initial set is composed with an atmospheric profile of temperature and water vapor from "Quebec meteorological center" fitted with local measurement (294.15 K for atmospheric ground temperature and $37 \%$ for relative humidity) and ground temperature obtained from the highest value of spectral brightness temperature measured on the scene. We use 150 tiny variations of atmospheric profiles and 150 different ground level temperatures to find the optimal set of parameters which leads to low spectral variation of retrieved spectral emissivity. For this concrete subimage the corresponding retrieved spectral emissivity is 


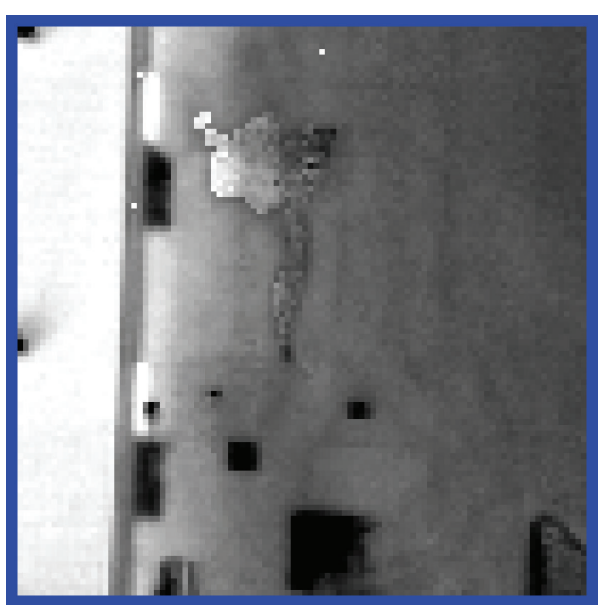

(a)

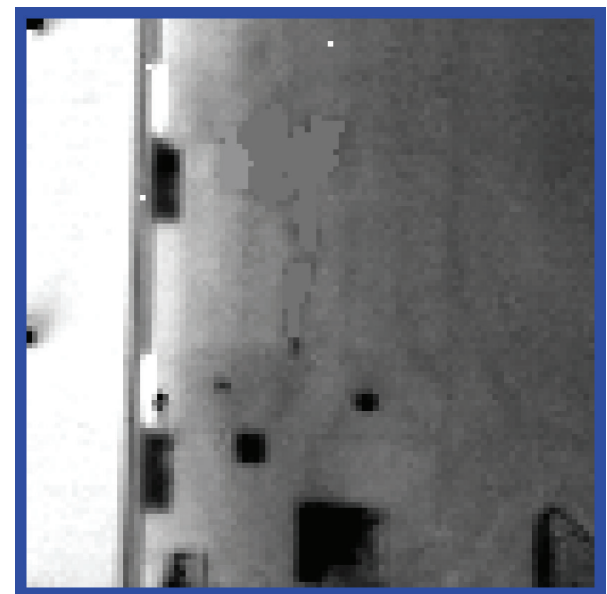

(b)

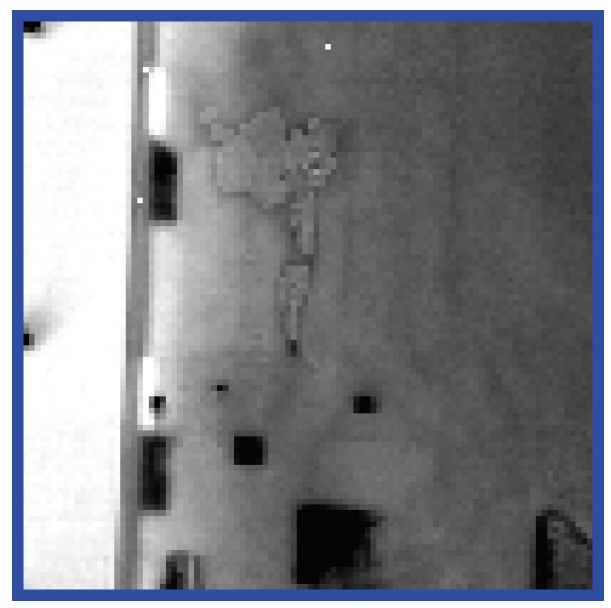

(c)

FIGURE 17: Comparison of the three background radiance estimations according to the band $950.4 \mathrm{~cm}^{-1}$, where the ethylene absorbance is the most important. (a) Selected-Band approach. (b) Clustering-Based method. (c) Clustering-Based Selected-Band method.

shown in Figure 18. The corresponding ground temperature, ground level atmospheric temperature, and water vapor concentration are, respectively, $316.5 \mathrm{~K}, 296.6 \mathrm{~K}$, and $10200 \mathrm{ppm}$.

From this atmospheric profile we compute atmospheric transmission and atmospheric radiance used for the atmospheric correction pixel by pixel.

Finally, the simple quantification algorithm used is from a linear model of the differential signature:

$$
\Delta \mathbf{L}=\mathbf{L}_{\text {on }}-\mathbf{L}_{\text {off }}=\rho \cdot \mathbf{A}(\lambda) \cdot\left(\mathbf{B}\left(\mathbf{T}_{\text {plume }}, \lambda\right)-\mathbf{L}_{\text {off }}\right),
$$

where $\mathbf{T}_{\text {plume }}$ is the plume temperature, $\rho$ is the column integrated concentration (ppm.m), and $\mathbf{B}(\mathbf{T}, \boldsymbol{\lambda})$ is the Planck function. One can estimate the concentration applying for each pixel by the following equation:

$$
\begin{aligned}
T & =\mathbf{A}(\boldsymbol{\lambda}) \cdot\left(\mathbf{B}\left(\mathbf{T}_{\text {plume }}, \boldsymbol{\lambda}\right)-\mathbf{L}_{\text {off }}\right), \\
\rho & =\frac{\mathbf{T}^{T} \boldsymbol{\Sigma}_{\mathbf{X}}^{-1} \Delta \mathbf{L}}{\mathbf{T}^{T} \boldsymbol{\Sigma}_{\mathbf{X}}^{-1} \mathbf{T}} .
\end{aligned}
$$

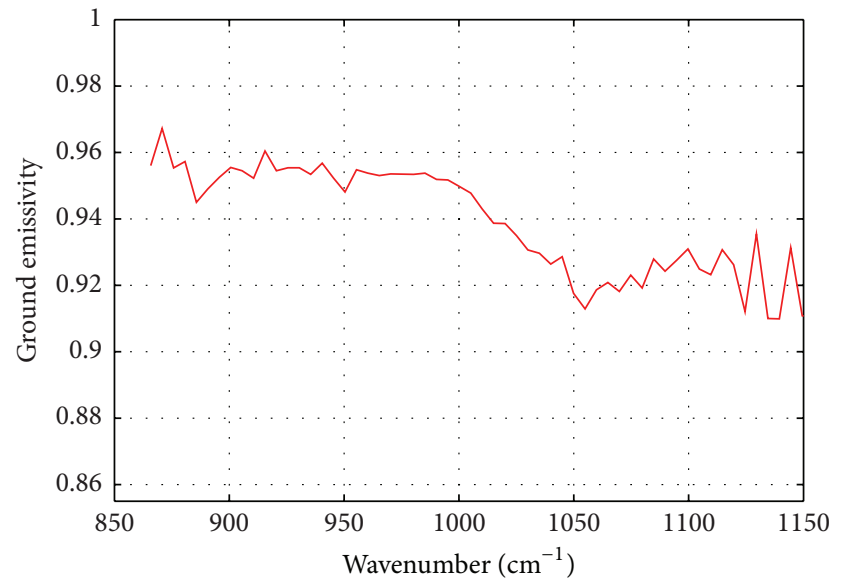

FIGURE 18: Mean ground spectral emissivity retrieved through the subimage.

Plume temperature is assumed to be homogeneous and equal to ground level atmospheric temperature estimated 


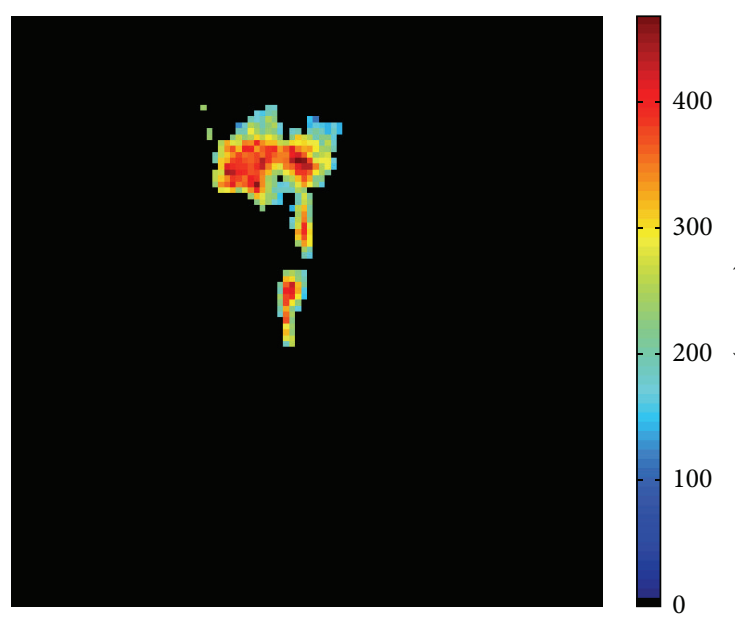

(a)

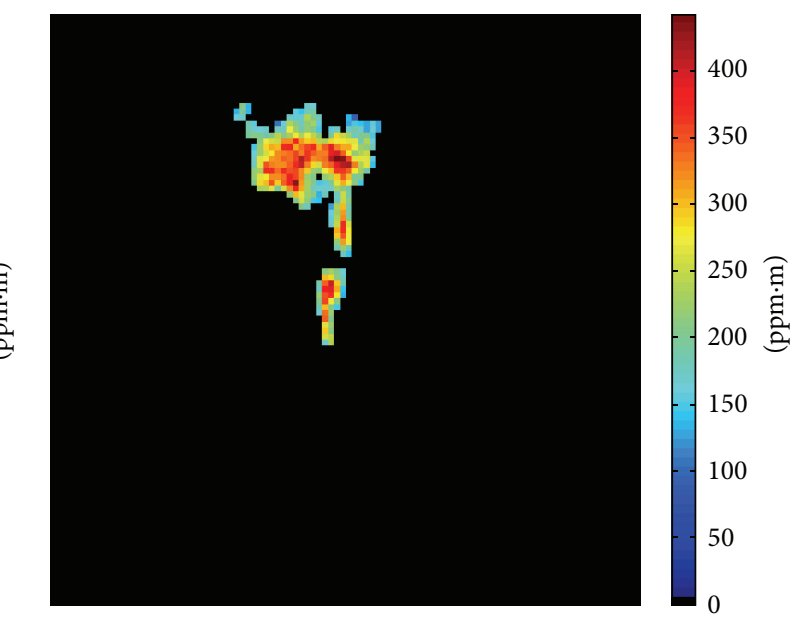

(b)

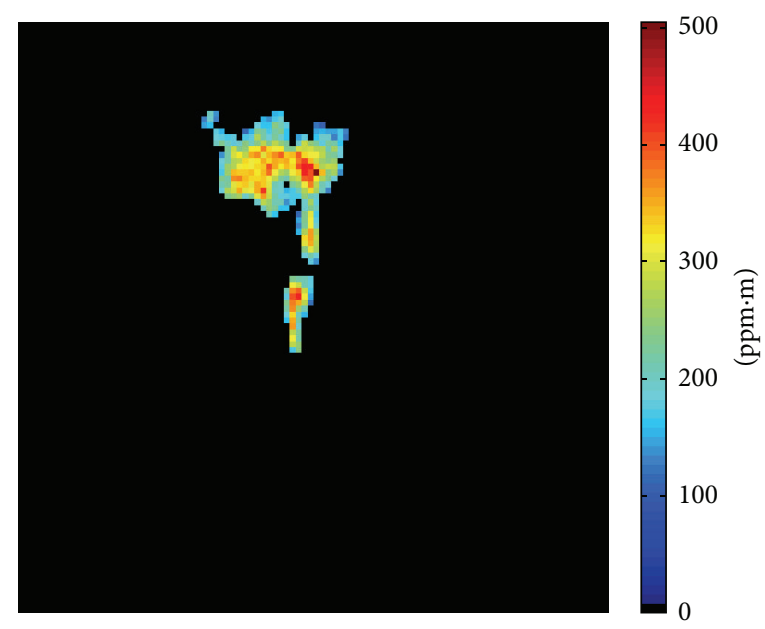

(c)

FIGURE 19: Retrieved ethylene concentration (ppm·m) from the three estimated $\mathbf{L}_{\text {off }}$ images. (a) Selected-Band approach. (b) Clustering-Based method. (c) Clustering-Based Selected-Band method.

to be $296.65 \mathrm{~K}$. One can notice than neither pixel-by-pixel emissivity nor ground temperature is needed here to estimate gas concentration.

The different stages described above are applied to the estimated $\mathbf{L}_{\text {off }}$ images shown in Figure 17.

Figures 20 and 19 shows ethylene concentration retrieved from Hyper-Cam data using the three estimated background methods outlined above and their differences. The mean retrieved integrated concentration is about $200 \mathrm{ppm}$ by CSB method. One can notice that ground radiance main differences are translated into retrieved concentration differences. In particular, the upper left corner of the plume where the background radiance was overestimated by about $10 \%$ by SB method leads to the highest differences in concentration (up to $200 \mathrm{ppm} \cdot \mathrm{m}$ ). Then, class borders used for CB method where radiance difference was the highest with CSB method lead to local differences of about $40 \mathrm{ppm} \cdot \mathrm{m}$. The mean difference between CB and CSB methods is less than $10 \%$ in concentration whereas it climbs up to $20 \%$ between SB and CSB methods. As pixel ground level size is about $23 \mathrm{~cm}$ the plume mean width size $l$ orthogonal to wind direction is calculated to $4 \mathrm{~m}$ and average mass in one-meter long slice of the plume is calculated to be $0.986 \mathrm{~g}$ assuming ethylene molar weight wm of $28 \mathrm{~g} / \mathrm{mol}$. The flow rate $d$ can be estimated to be $4.2 \mathrm{~g} / \mathrm{s}$ assuming a wind speed value of $4.3 \mathrm{~m} / \mathrm{s}$ and using the following equation where $\rho$ (in ppm.m) is the mean integrated column concentration in an orthogonal slice of one meter thick and $4 \mathrm{~m}$ width. This results fits well with the true value of $20 \mathrm{~L} / \mathrm{min}$ which corresponds to $3.15 \mathrm{~g} / \mathrm{s}$ assuming that ethylene volume weight is $567.65200 \mathrm{~kg} / \mathrm{m}^{3}$ :

$$
d=\rho \cdot l \cdot 10^{3} \cdot \frac{w m}{22.71} \text {. }
$$

\section{Summary and Conclusions}

Gas trace plume remote sensing above industrial scene requires both high spectral and spatial resolution. Using airborne FTS imaging techniques it becomes possible to detect and quantify gas traces. However retrieval accuracy depends on both pixel-by-pixel background under plume 


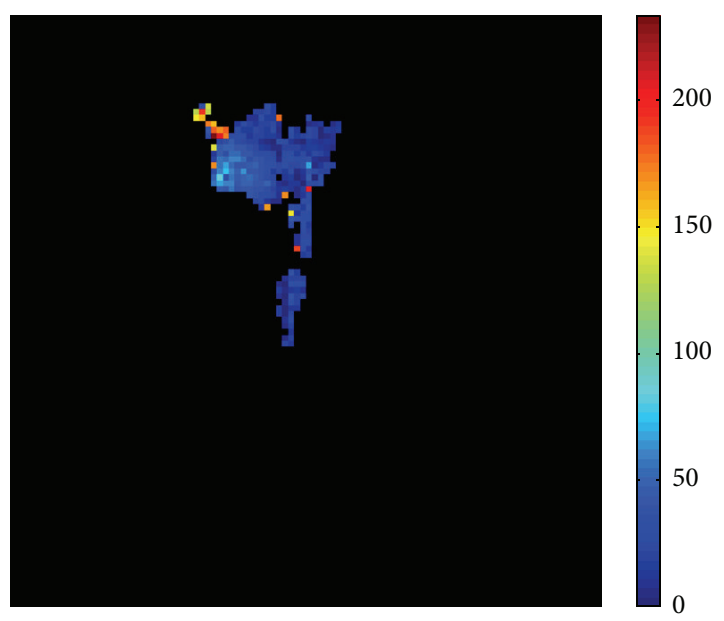

(a)

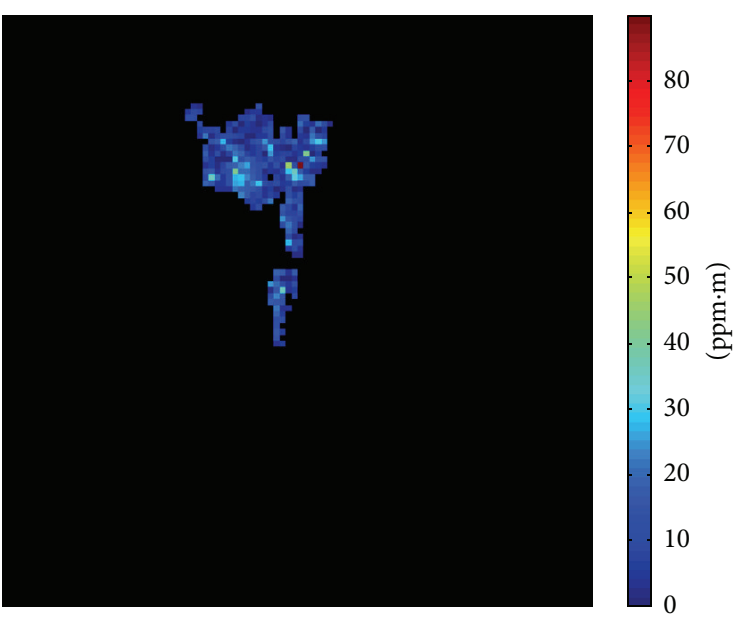

(b)

FIGURE 20: Comparison between retrieved concentration from the different approaches. (a) Difference between retrieved concentration from SB background estimation and CSB background estimation. (b) Difference between retrieved concentration from CB background estimation and CSB background estimation.

estimation and good enough spectral resolution around target gas absorption peak. In this paper we propose a method to estimate ground radiance pixel by pixel taking into account scene variability spectral behavior. The CSB method described above is a Clustering-Based method which aims to associate statistical spectral properties from classes nonimpacted by the plume to classes below the target gas plume. This method is based on SB method algorithm [18] extended to a clustering approach. We have shown that introducing an initial classification of the scene improves background radiance estimation for nonhomogeneous scene in comparison with classical SB method or simple ClusteringBased method. Results based on simulations show more than $10 \mathrm{~K}$ in brightness temperature for low emissivity material and up to $3 \mathrm{~K}$ for high emissivity material.

The CSB method applied to airborne Hyper-Cam LW data acquired above an ethylene release of $20 \mathrm{~L} / \mathrm{min}$ shows that the CSB method can reduce background radiance uncertainty from 5 to $1 \%$ or from 3 to $1 \mathrm{~K}$ in terms of brightness temperature uncertainty, for a high emissivity materiel such as concrete. This improvement leads to a mean difference of about $10 \%$ on ethylene plume retrieved concentrations in this particular "easy" scene.

Finally we propose in this paper an end-to-end method using a single hyperspectral image to estimate (i) ground radiance and properties (emissivity and temperature); (ii) atmospheric ground level temperature and water vapor content; and (iii) trace gas plume concentration with an encouraging accuracy. Indeed the retrieved flow rate is close to mean flow rate during the gas release.

The method outlined above must now be tested on industrial scenes where expected improvement should be higher as it deals well with a very high number of materials, with intraclass variability such as shadow and temperature gradient and with low emissivity materials.

\section{Symbols}

\section{Plume Detection}

$N$ : Number of pixels

$N_{\mathrm{B}}$ : Number of spectral bands

$\mathbf{X}$ : Hyperspectral image matrix

$\left[N \times N_{\mathrm{B}}\right] . \mathrm{X}$ could represent radiance

(L) or brightness temperature $\left(T_{\mathrm{b}}\right)$

$\mathbf{x}$ : A pixel of $\mathbf{X}$

$\langle\mathbf{x}\rangle$ : Mean spectrum of $\mathbf{X}$

$\Sigma_{\mathbf{X}}$ : Covariance of $\mathbf{X}$

T: The gaseous target spectrum

$\boldsymbol{\alpha}$ : Target fit coefficients (abundances)

d: Residual vector.

\section{Selected-Band Approach}

$N_{\text {on }}$ : Number of plume-present pixels

$N_{\text {off }}$ : Number of plume-absent pixels

$N_{\mathrm{SB}}$ : Number of selected bands

$N_{\mathrm{p}}$ : $\quad$ Number of the used principal components

$L_{\text {on }}(\lambda)$ : On-plume radiance: measured radiance for a plume-present pixel

$L_{\text {off }}(\lambda)$ : Off-plume radiance: measured radiance for a plume-absent pixel

$L_{\text {bkg }}(\lambda)$ : Background (plume-free) radiance for a pixel.

\section{Classification}

$D_{\max }$ : Threshold on the size of a class

$N_{\mathrm{C}}$ : Number of classes without plume

$N_{\mathrm{CP}}$ : Number of classes containing plume. 


\section{Competing Interests}

The authors declare that they have no competing interests.

\section{References}

[1] M. Schlerf, G. Rock, P. Lagueux et al., "A hyperspectral thermal infrared imaging instrument for natural resources applications," Remote Sensing, vol. 4, no. 12, pp. 3995-4009, 2012.

[2] J. A. Hackwell, D. W. Warren, R. P. Bongiovi et al., "LWIR/MWIR imaging hyperspectral sensor for airborne and ground-based remote sensing," in Imaging Spectrometry II, vol. 2819 of Proceedings of SPIE, pp. 102-107, Denver, Colo, USA, November 1996.

[3] Y. Ferrec, S. Thétas, J. Primot et al., "Sieleters, an airborne imaging static fourier transform spectrometer: design and preliminary laboratory results," in Proceedings of the Imaging and Applied Optics Congress, Paper FM1D-3, OSA, Arlington, Va, USA, June 2013.

[4] D. W. Messinger, S. S. Shen, and P. E. Lewis, "Gaseous plume detection in hyperspectral images: a comparison of methods," in Proceedings of the SPIE, Algorithms and Technologies for Multispectral, Hyperspectral, and Ultraspectral Imagery X, vol. 5425, pp. 592-603, Orlando, Fla, USA, August 2004.

[5] L. Chilton and S. Walsh, "Detection of gaseous plumes using basis vectors," Sensors, vol. 9, no. 5, pp. 3205-3217, 2009.

[6] E. Hirsch and E. Agassi, "Detection of gaseous plumes in IR hyperspectral images using hierarchical clustering," Applied Optics, vol. 46, no. 25, pp. 6368-6374, 2007.

[7] C. C. Funk, J. Theiler, D. A. Roberts, and C. C. Borel, "Clustering to improve matched filter detection of weak gas plumes in hyperspectral thermal imagery," IEEE Transactions on Geoscience and Remote Sensing, vol. 39, no. 7, pp. 1410-1420, 2001.

[8] A. K. Thorpe, D. A. Roberts, P. E. Dennison, E. S. Bradley, and C. C. Funk, "Point source emissions mapping using the airborne visible/infrared imaging spectrometer (AVIRIS)," in Proceedings of the 18th Annual Conference on Algorithms and Technologies for Multispectral, Hyperspectral, and Ultraspectral Imagery, vol. 8390, Baltimore, Md, USA, April 2012.

[9] C. M. Gittins, "Detection and characterization of chemical vapor fugitive emissions by nonlinear optimal estimation: theory and simulation," Applied Optics, vol. 48, no. 23, pp. 45454561, 2009.

[10] N. B. Gallagher, B. M. Wise, and D. M. Sheen, "Estimation of trace vapor concentration-pathlength in plumes for remote sensing applications from hyperspectral images," Analytica Chimica Acta, vol. 490, no. 1-2, pp. 139-152, 2003.

[11] S. J. Young, "Detection and quantification of gases in industrialstack plumes using thermal-infrared hyperspectral imaging," Tech. Rep. ATR-2002(8407)-1, The Aerospace Corporation, 2002.

[12] C. S. Turcotte and M. R. Davenport, "Gas plume quantification in downlooking hyperspectral longwave infrared images," in Image and Signal Processing for Remote Sensing XVI, vol. 7830 of Proceedings of SPIE, Toulouse, France, September 2010.

[13] P. Heasler, C. Posse, J. Hylden, and K. Anderson, "Nonlinear Bayesian algorithms for gas plume detection and estimation from Hyper-spectral thermal image data," Sensors, vol. 7, no. 6, pp. 905-920, 2007.

[14] S. Walsh, L. Chilton, M. Tardiff, and C. Metoyer, "Effect of the temperature-emissivity contrast on the chemical signal for gas plume detection using thermal image data," Sensors, vol. 8, no. 10, pp. 6471-6483, 2008.

[15] N. B. Gallagher, B. M. Wise, and D. M. Sheen, "Error analysis for estimation of trace vapor concentration pathlength in stack plumes," Applied Spectroscopy, vol. 57, no. 6, pp. 614-621, 2003.

[16] S. E. Golowich and D. G. Manolakis, "Cramer-Rao bounds for long-wave infrared gaseous plume quantification," Optical Engineering, vol. 53, no. 2, 2014.

[17] J. Theiler, B. R. Foy, and A. M. Fraser, "Nonlinear signal contamination effects for gaseous plume detection in hyperspectral imagery," in Defense and Security Symposium, vol. 6233 of Proceedings of SPIE, 2006.

[18] S. Niu, S. E. Golowich, V. K. Ingle, and D. G. Manolakis, "New approach to remote gas-phase chemical quantification: selected-band algorithm," Optical Engineering, vol. 53, no. 2, Article ID 021111, 2014.

[19] S. W. Sharpe, R. L. Sams, T. J. Johnson, P. M. Chu, G. C. Rhoderick, and F. R. Guenther, "Creation of $0.10 \mathrm{~cm}^{-1}$ resolution, quantitative, infrared spectral libraries for gas samples," in Vibrational Spectroscopy-Based Sensor Systems, vol. 4577 of Proceedings of SPIE, pp. 12-24, Boston, Mass, USA, October 2001.

[20] S. W. Sharpe, T. J. Johnson, R. L. Sams, P. M. Chu, G. C. Rhoderick, and P. A. Johnson, "Gas-phase databases for quantitative infrared spectroscopy," Applied Spectroscopy, vol. 58, no. 12, pp. 1452-1461, 2004.

[21] A. Hayden, E. Niple, and B. Boyce, "Determination of trace-gas amounts in plumes by the use of orthogonal digital filtering of thermal-emission spectra," Applied Optics, vol. 35, no. 16, pp. 2802-2809, 1996.

[22] A. M. Baldridge, S. J. Hook, C. I. Grove, and G. Rivera, "The ASTER spectral library version 2.0," Remote Sensing of Environment, vol. 113, no. 4, pp. 711-715, 2009.

[23] V. Masson, L. Gomes, G. Pigeon et al., “The Canopy and Aerosol Particles Interactions in TOulouse Urban Layer (CAPITOUL) experiment," Meteorology and Atmospheric Physics, vol. 102, no. 3-4, pp. 135-157, 2008.

[24] C. Miesch, L. Poutier, V. Achard, X. Briottet, X. Lenot, and Y. Boucher, "Direct and inverse radiative transfer solutions for visible and near-infrared hyperspectral imagery," IEEE Transactions on Geoscience and Remote Sensing, vol. 43, no. 7, pp. 1552-1561, 2005.

[25] D. J. Carruthers, R. J. Holroyd, J. C. R. Hunt et al., "UKADMS: a new approach to modelling dispersion in the earth's atmospheric boundary layer," Journal of Wind Engineering and Industrial Aerodynamics, vol. 52, pp. 139-153, 1994.

[26] J. R. Schott and C. Y. Chang, "Synthetic image generation of chemical plumes for hyperspectral applications," Optical Engineering, vol. 39, no. 4, pp. 1047-1056, 2000.

[27] J. R. Landis and G. G. Koch, "The measurement of observer agreement for categorical data," Biometrics, vol. 33, no. 1, pp. 159-174, 1977.

[28] C. C. Borel, "Surface emissivity and temperature retrieval for a hyperspectral sensor," in Proceedings of IEEE Conference on Geoscience and Remote Sensing, pp. 504-509, IEEE, July 1998.

[29] K. Kanani, L. Poutier, F. Nerry, and M. P. Stoll, "Directional effects consideration to improve out-doors emissivity retrieval in the 3-13 $\mu \mathrm{m}$ domain," Optics Express, vol. 15, no. 19, pp. 12464-12482, 2007. 

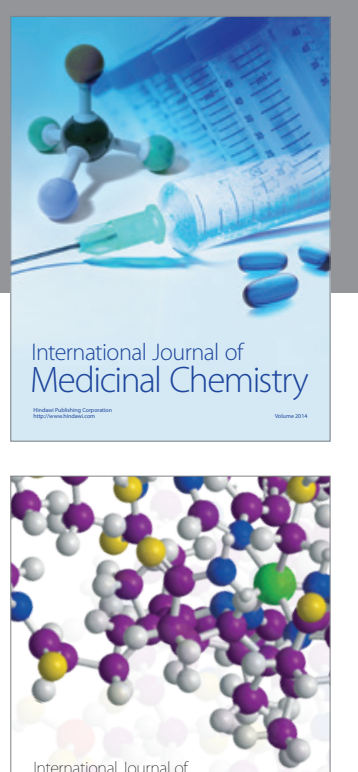

Carbohydrate Chemistry

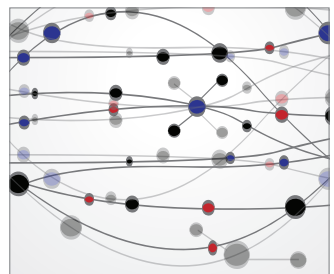

The Scientific World Journal
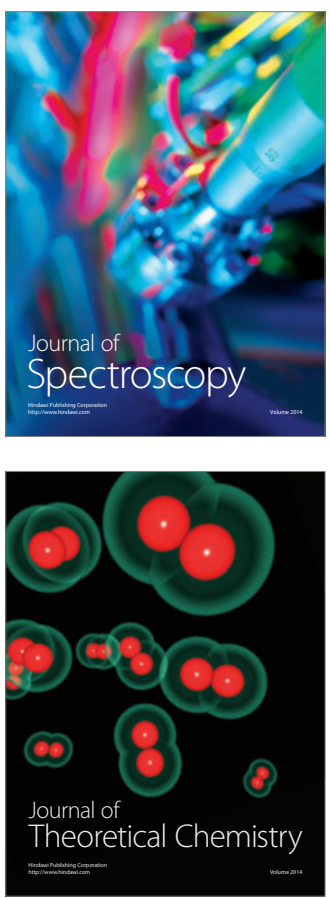
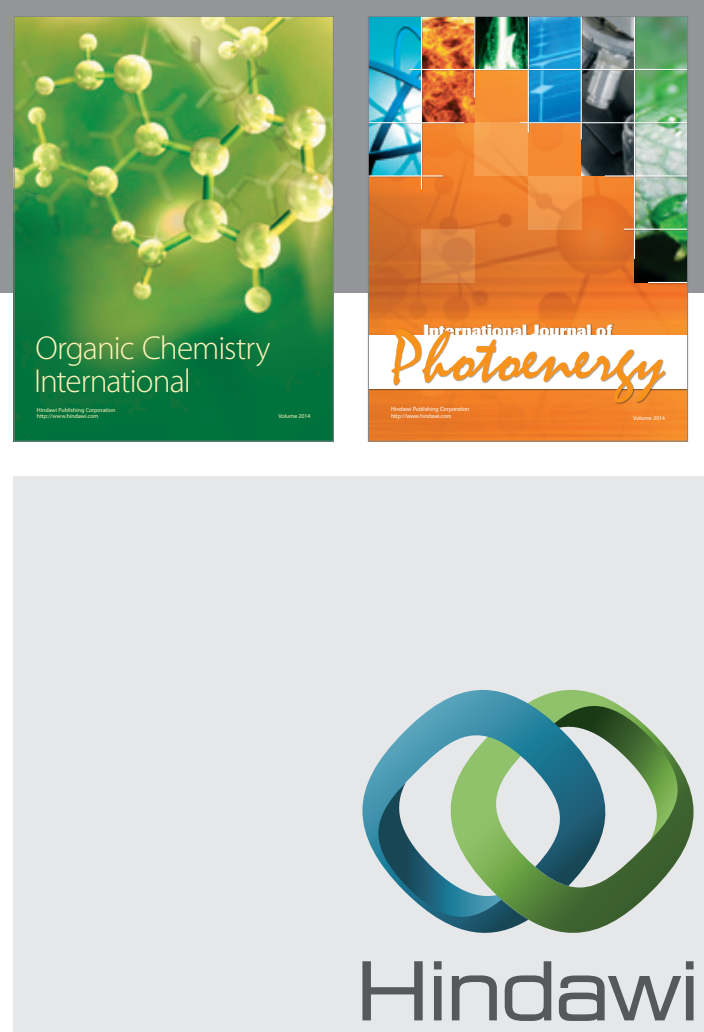

Submit your manuscripts at

http://www.hindawi.com

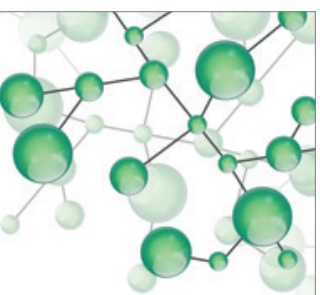

International Journal of

Inorganic Chemistry

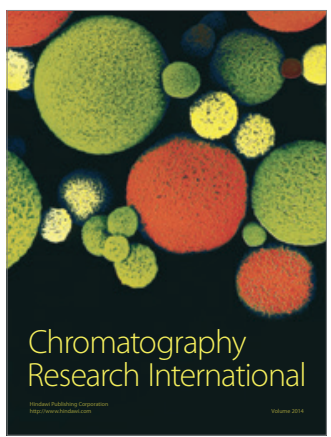

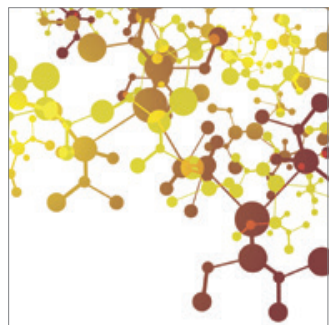

Applied Chemistry
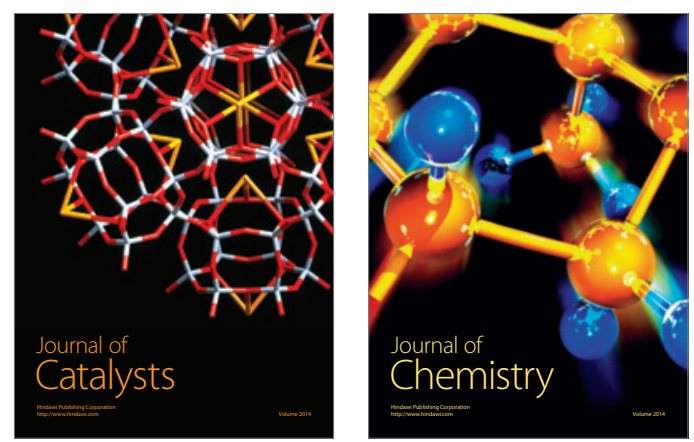
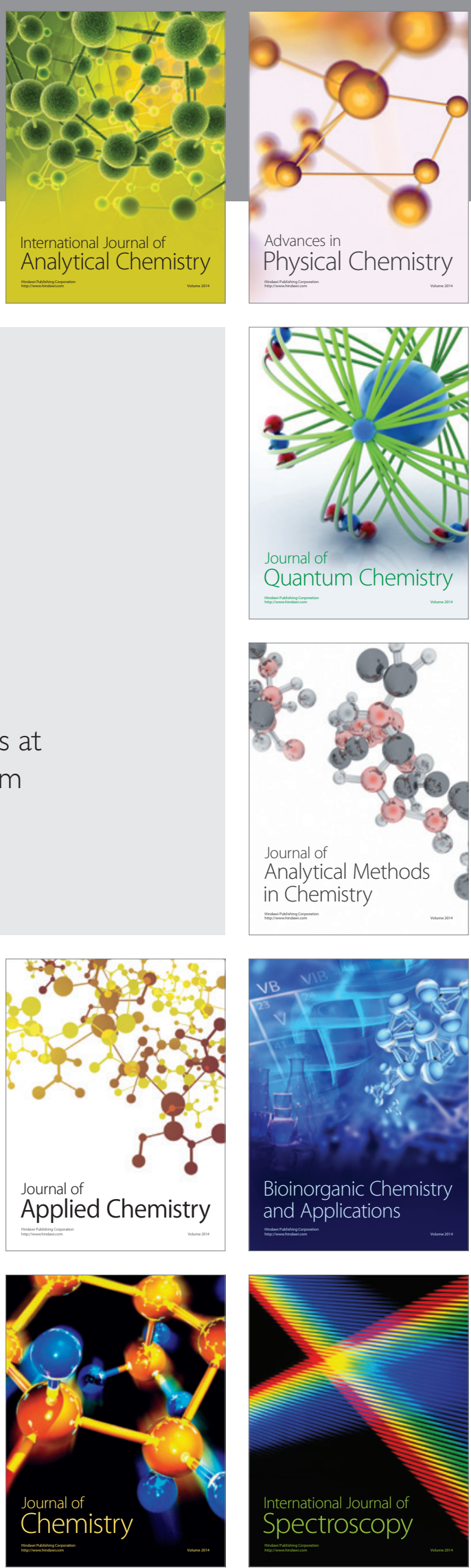